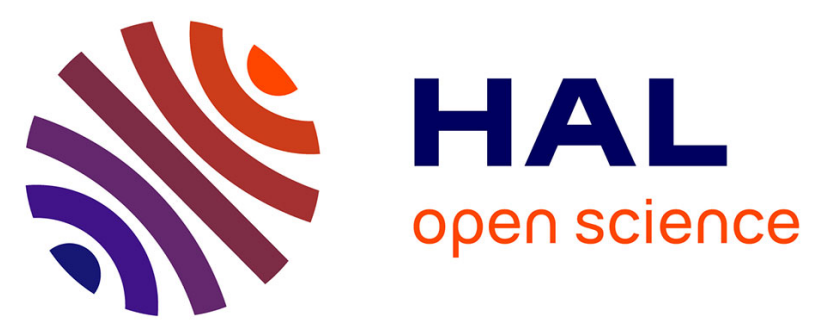

\title{
Prediction and analysis of quasi-periodic solution for friction-induced vibration of an industrial brake system with the Generalized Modal Amplitude Stability Analysis
}

\author{
Enora Denimal, Jean-Jacques Sinou, S. Nacivet
}

\section{To cite this version:}

Enora Denimal, Jean-Jacques Sinou, S. Nacivet. Prediction and analysis of quasi-periodic solution for friction-induced vibration of an industrial brake system with the Generalized Modal Amplitude Stability Analysis. Journal of Sound and Vibration, 2021, pp.116164. 10.1016/j.jsv.2021.116164 . hal-03214459

\author{
HAL Id: hal-03214459 \\ https://hal.science/hal-03214459
}

Submitted on 1 May 2021

HAL is a multi-disciplinary open access archive for the deposit and dissemination of scientific research documents, whether they are published or not. The documents may come from teaching and research institutions in France or abroad, or from public or private research centers.
L'archive ouverte pluridisciplinaire HAL, est destinée au dépôt et à la diffusion de documents scientifiques de niveau recherche, publiés ou non, émanant des établissements d'enseignement et de recherche français ou étrangers, des laboratoires publics ou privés. 


\title{
Prediction and analysis of quasi-periodic solution for friction-induced vibration of an industrial brake system with the Generalized Modal Amplitude Stability Analysis
}

\author{
Enora Denimal ${ }^{1,2,{ }^{*}}$, Jean-Jacques Sinou ${ }^{2,3}$, and Samuel Nacivet ${ }^{2}$ \\ ${ }^{1}$ Laboratoire de Tribologie et Dynamique des Systèmes, UMR CNRS 5513, École Centrale de Lyon, \\ 36 avenue Guy de Collongue 69134 Écully Cedex, France \\ ${ }^{2}$ PSA Peugeot Citroën, Centre technique de la Garenne Colombes, 18 rue des Fauvelles, \\ 92250 La Garenne Colombes, France \\ ${ }^{3}$ Institut Universitaire de France, 75005 Paris, France \\ *Corresponding author: enora.denimal@inria.fr
}

\begin{abstract}
Brake squeal is a major issue for car manufacturers as it is the reason for the return of many vehicles to customer services, representing high costs for the companies. To meet customer's expectations, squeal must be accurately predicted during the design process. In the context of the automotive industry, squeal usually refers to friction-induced vibrations that generate noise. The main methodology employed nowadays for friction-induced vibrations prediction of industrial systems is the well-known complex eigenvalue analysis (CEA) despite its limitations. The latter suffers from an under- or over-predictive aspect and the vibration amplitudes cannot be estimated. A recent approach, called the generalised modal amplitude stability analysis (GMASA), has been developed as a complementary approach of the CEA to identify the modes involved in the nonlinear dynamic response of systems subjected to friction-induced vibrations and to approximate the quasi-periodic oscillations. The objective of this paper is to predict the nonlinear dynamic response of a full industrial automotive brake system. The GMASA is employed to predict its nonlinear dynamic response. It is demonstrated that when the CEA predicts a single unstable mode, two are actually involved in the nonlinear dynamic response. The quasi-periodic oscillations are analysed, as well as the evolution of the contact conditions at the pad/disc interface and exhibits the presence of micro-impacts.
\end{abstract}

Keywords Friction-induced vibrations, squeal, finite element model, generalised modal amplitude stability analysis, contact, friction, multi-instabilities, quasi-periodic

\section{Introduction}

Car manufacturers are leading research and development to meet environmental and quality customers' expectations. Customer satisfaction surveys show that a large part of warranty claims is due to friction-induced vibration and squeal noise. Even if the main function of a brake system is of safety order and if squeal has no impact on the braking quality, this friction noise is nasty as it can reach high sound levels up to $110 \mathrm{~dB}$ and is often associated to a brake system failure in the collective imagination. In consequence, squeal noise is at the origin of a large part of warranty claims representing high costs for the automotive industry. Therefore, friction-induced vibration and noise have become a priority topic over the last decades for the automotive industry, where more and more constraints on friction noise must be meet. This justifies the need to predict friction-induced vibrations early in the design stage.

Friction-induced vibrations have been extensively studied over the last decades by academics and industrials from two complementary points of view, a purely tribological one [1 6 and from a structural dynamic one $7-18$. The prediction of the friction-induced vibrations is made complex by its fugitive aspect and its sensitivity to many parameters [19, 20] as the environment [21], the wear [22, the material [23, internal contact conditions [24] or the friction contact law 25, 26]. These observations have pushed researchers to even consider this sensitivity as an intrinsic part of the appearances of unstable modes and friction-induced vibrations [25, 26] and to consider this phenomenon with its variable aspect to improve prediction of unstable modes and friction-induced vibration and noise $[27[31]$. 
Despite the numerous studies on the topic, the emergence and the phenomenon at the origin friction-induced vibrations are still not well understood. If some unstable modes are at the origin of the initial vibrations, other modes can be present in the final steady-state response [8, 32, and even if the system is vibrating, noise is not necessarily present. In 33, Oberst and Lai observed some chaotic behaviours during friction-induced vibrations, however (quasi)-periodic motions were present in majority. In [32, 34, Wernitz and Hoffmann observed chaotic nature of the dynamic before the appearance of unstable behaviour and the associated friction-induced vibration and state that the observed phenomena was not the result of a system loosing its stability to a quasi-periodic solution, but the result of a chaotic attractor approaching a regular attractor [35, 36. In spite of these analysis, once the phenomena of friction-induced vibration is present, (quasi)-periodic solutions are most of the time observed [17, 29, 33.

The numerical prediction of squeal can be split into two different parts, first the prediction of the frictioninduced vibrations, and second the computation of the transmitted acoustic noise. For the second aspect, the reader is invited to refer to [37-40] for more details as it is not in the scope of the present study. Considering the prediction of friction-induced vibrations, the most employed method in the industry remains the Complex Eigenvalue Analysis (CEA) [8, 9, 37]. It consist in the computation of the complex modes of the brake system around its nonlinear static equilibrium. If this method is robust and easy to implement even for large Finite Element Models (FEM) of brake systems, it is well-known that is has an over- or under-predictive aspects [8, 9]. In other words, the mode predicted as unstable by the CEA are not necessarily responsible for the frictioninduced vibrations, and on the opposite, modes that can be responsible for friction-induced vibrations are not predicted as unstable by the CEA. Several reasons explain this limitation, the first one being that the CEA is a linear approach that doesn't account for the nonlinear contributions responsible for friction-induced vibrations, and the second being modelling errors and/or uncertainties, more especially related to the contact [25, 26, 29, 37].

To counterbalance the prediction limitations of the CEA, different numerical strategies have been investigated to predict friction-induced vibrations. First of all, time integration has been considered to get the transient regime and the stationary response without any hypothesis on the form of the nonlinear response [11, 12, 32, 41, 42. Through this approach, the history of the system and the different modal interactions of the transient part can be analysed. Yet, temporal integration represents an extremely prohibitive numerical cost which makes this approach unusable in an industrial context. Some works have focused on the computation of the stationary response directly to reduce the computational cost. Hence, in [43. Coudeyras et al. proposed the constrained harmonic balance method (CHBM). The vibrations frequencies of the system (that are unknown) are added in the HBM formulation through an additional constraint. If interesting results were obtained, the initialisation and the convergence of the method remains tedious and limit the interest of the approach. In [44, Charroyer et al. proposed an adaptation of the shooting method where an energetic criterion is developed to initialise the method. Nevertheless, no extension to the multi-instability case have been developed so far. More recent strategies intend to complete the CEA by considering additional criterion. In [45], Brunetti et al. defined a Modal Absorption Index (MAI) that characterizes the capacity of each mode to absorb energy and to become unstable. In [46, Nacivet and Sinou developed the Modal Amplitude Stability Analysis (MASA) to predict unstable vibration mode when only one is present. The dynamic response is approximated based on a linearisation of the initial dynamic response around a nonlinear sliding equilibrium position for a given modal amplitude. In [47, the authors extended this method to the multi-instability case, called Generalised Modal Amplitude Stability Analysis (GMASA), and demonstrated the validity of the approach on a phenomenological model.

The objective of the present study is to demonstrate that the GMASA method is able to compensate the under-predictive limitation of the CEA by predicting the quasi-periodic solution of an industrial FEM of a brake system and by capturing the emergence of a second unstable mode in the nonlinear dynamic response. For the first time, the GMASA is applied on a full FEM of an industrial brake for the treatment of multiinstability. The GMASA is based on successive linearisations of the nonlinear dynamic response where nonlinear contributions are integrated as contributions in the brake structural matrices. The possible coupling between the different unstable modes is possible and done through the computation of the nonlinear forces with an AFT approach. Numerically speaking, successive stability analysis are performed where the nonlinear contribution are accounted for, which make it robust and efficient for the study of industrial brake systems, and compensate the first limitation of the CEA. As explained previously, experimental studies indicate that when the steadystate regime is reached, (quasi)-periodic solutions are observed, which justifies the choice of an approach as the GMASA to predict the unstable vibration modes. Results show that when a Coulomb's friction law is used as a tangential friction law, the appearance of impacts at the pad/disc interface can be enough to stabilise the amplitude of the oscillations. Moreover, if only one mode was unstable initially, the growth of amplitude and the coupling between the different modes due to the nonlinear forces may be responsible for the emergence of another fundamental contribution (i.e. a new unstable mode) in the nonlinear dynamic response. The appearance of 


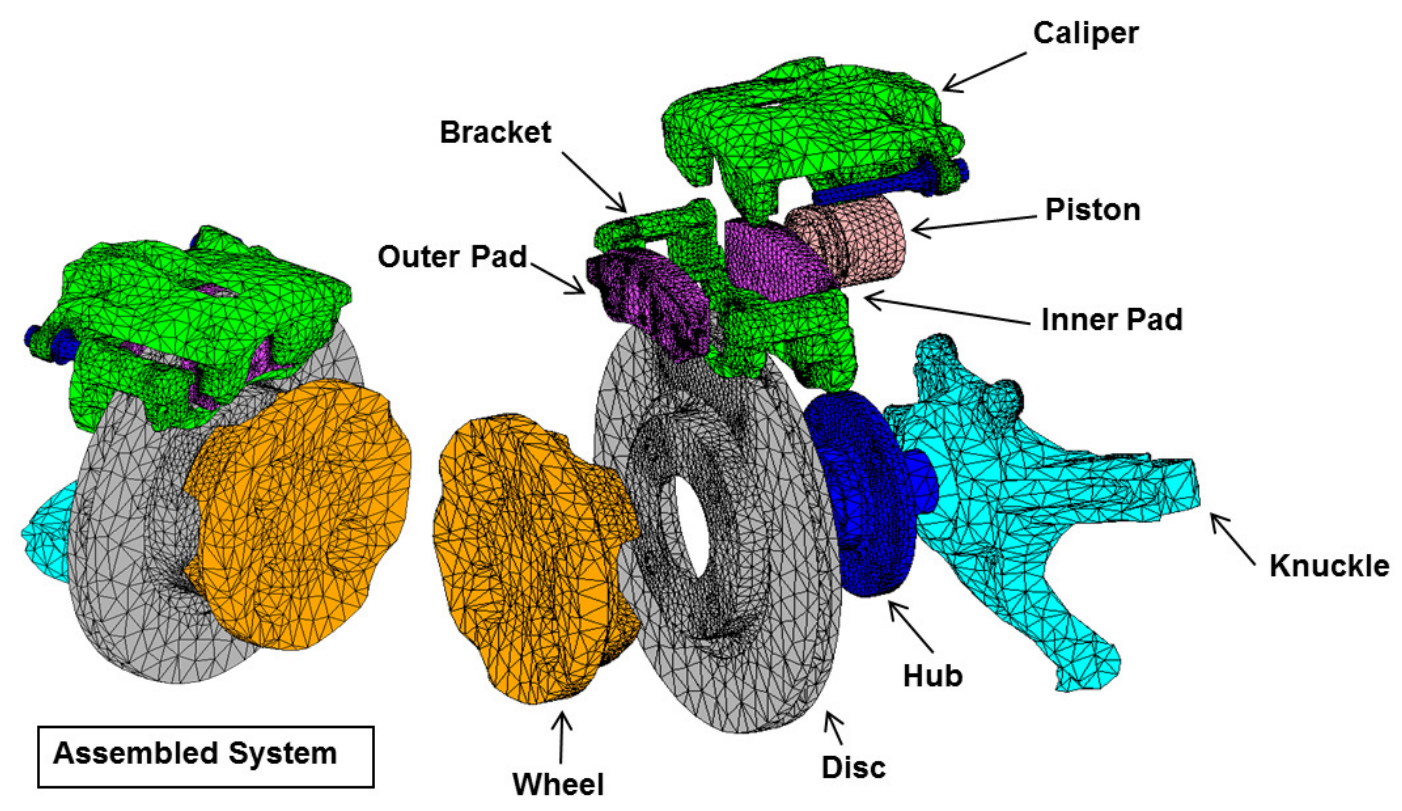

Figure 1: Finite Element Model of the brake system: assembled (left) and exploded (right) views

this second unstable mode and its coexistence with the first unstable mode may lead to more complex solutions corresponding to torus motion and quasi-periodic oscillations, and consequently different vibrational behaviours depending on the contact point.

The paper follows the following structure, first the FEM of the automotive brake system is presented. Second, the stability analysis is briefly recalled. Third, the GMASA approach is explained in details and finally in the fifth section it is applied on the FEM of the brake system to predict its dynamic response and results are analysed.

\section{System presentation}

The model under study is a FEM of an automotive brake system, shown in Figure 1 . The latter is composed of a disc, two pads, a bracket, a caliper, a piston, a knuckle and a wheel. It is a floating caliper technology. During a braking action, the hydraulic pressure pushes the piston against the inner pad. The reaction forces pull the caliper back, dragging the outer pad with it, which finally enters in contact with the disc. This action creates friction between the disc and the pads, dissipating energy. Sometimes, this is also accompanied by squeal noise. If the latter has no effect on braking efficiency, it is often associated with a brake failure in the collective imagination, justifying the return of many vehicles to the customer service. To avoid this cost, the prediction of friction-induced vibrations and noise is of prior importance in the automotive industry.

The squeal noise originates from vibrations of the brake system due to instability and mode coupling between the different components of the brake system. Traditionally, the friction coefficient at the pad/disc interface is considered as the most influential parameter on the automotive brake system stability and usually the propensity of unstable modes of a brake system is studied for different values of this friction coefficient during the design process. It has been shown recently that the contact between the different components, and more specifically the contact between the pads and the other components, have a strong influence on the brake stability 24 .

The FEM is implemented in Abaqus, and a volume mesh of 103,444 elements and 182,337 nodes is created with 10-node quadratic tetrahedron elements. The FEM has been validated with a convergence study with regards to the eigenvalues and mode shapes [48. The different material properties of the different components are presented in Table 1. The brake lining is an orthotropic material, each line corresponds to the material properties in one dimension. The braking pressure as well as the disc rotation velocity are constant. The hydraulic pressure action is modelled by an effort to the back of the piston.

To reduce the computational cost associated to the stability analysis, an implicit reduction technique that is based on a nodal reduction at the frictional interface and a substructure generation at the nonlinear static equilibrium is employed, as detailed in [48. With this approach, the pad/disc interface is reduced to a small number of contact points used for a node-to-node contact formulation. Moreover, a classical Craig-Bampton reduction is also applied, where the reduced number of nodes at the pad/disc interface are used as reduction 


\begin{tabular}{lllll}
\hline Element & Material & Young Modulus (GPa) & Poisson's coefficient & Density $\left(\mathbf{k g} / \mathbf{m}^{3}\right)$ \\
\hline Disc & Grey cast iron & 113 & 0.275 & 7075 \\
Caliper & Ductile cast iron & 175 & 0.275 & 7200 \\
Bracket & Ductile cast iron & 175 & 0.275 & 7200 \\
Pad (support) & Steel & 210 & 0.29 & 7800 \\
Brake lining & GA7504 & 10.66 & 0.09 & 4870 \\
& & 10.66 & 0.14 & \\
\hline
\end{tabular}

Table 1: Material properties of the brake components

nodes. The full process for the generation of each substructure and of the brake assembly as well as the validity of the reduced FEM versus the mesh quality and the number of interface nodes have been previously explained in [48].

The resulting dynamic equation of the reduced brake system is given by:

$$
\mathbf{M U ̈}+\mathbf{C} \dot{\mathbf{U}}+\mathbf{K}_{n l} \mathbf{U}+\mathbf{F}_{\mathbf{n l}}(\mathbf{U})=\mathbf{F}_{\text {ext }}
$$

where $\mathbf{M}$ and $\mathbf{C}$ are the mass and damping matrices respectively. $\mathbf{F}_{\text {ext }}$ is the vector of the exterior forces applied on the brake, here the braking pressure. $\mathbf{F}_{\mathbf{n l}}$ is the vector of the nonlinear contact and frictional forces at the pad/disc contact interfaces. Based on experimental data [43, the normal contact force $F_{N}$ is modelled with a linear and a cubic terms when there is contact. The formulation is:

$$
F_{N}\left(\delta_{n}\right)=\left\{\begin{array}{l}
k_{l} \delta_{n}+k_{n l} \delta_{n}^{3} \text { if } \delta_{n} \geq 0 \\
0 \text { otherwise }
\end{array}\right.
$$

with $\delta_{n}$ the relative displacement at the contact point $n, k_{l}$ the linear contact stiffness and $k_{n l}$ the nonlinear contact stiffness. This node-to-node formulation is possible thanks to the nodal reduction technique proposed in [48. The classical Coulomb's law is used for the friction law. $\mathbf{K}_{n l}$ is the stiffness matrix constructed from the structural stiffness matrix of the different components and from the contribution of the different contact interfaces between the pads and the piston, the pads and the bracket and the pads and the caliper. These internal contacts have a strong influence on the propensity of unstable modes 24. This matrix is defined as:

$$
\mathbf{K}_{\text {nl }} \mathbf{U}=\mathbf{K} \mathbf{U}+\mathbf{F}_{\text {pad } / \text { piston }}(\mathbf{U})+\mathbf{F}_{\text {pad/bracket }}(\mathbf{U})+\mathbf{F}_{\text {pad/caliper }}(\mathbf{U})
$$

where $\mathbf{K}$ is the structural stiffness matrix, and the $\mathbf{F}_{i / j}$ are the equivalent stiffness contributions of the interfaces between the components $i$ and $j$ 24]. Two contact status are possible for each of these interfaces: contact or non-contact.

\section{Preamble: Stability analysis of the brake system}

First, a stability analysis for different values of the friction coefficient $\mu$ between the pads and the disc is performed to investigate the stability of the nonlinear static equilibrium position of the brake system, and to identify the modes of the system that might be unstable and so might be at the origin of the friction-induced vibrations. As a reminder, the stability analysis, based on the CEA, is reminded here. As it is the starting point of the GMASA method, the CEA is fully described.

\subsection{Complex Eigenvalue Analysis}

The nonlinear static equilibrium position $\mathbf{U}_{\mathbf{S}}$ of the system is determined by solving the equation $\mathbf{K}_{n l} \mathbf{U}_{\mathbf{S}}+$ $\mathbf{F}_{\mathbf{n l}}\left(\mathbf{U}_{\mathbf{S}}\right)=\mathbf{F}_{\text {ext }}$. The stability of this equilibrium position is then determined by considering a small perturbation $\boldsymbol{\Delta} \mathbf{U}$ around this position, in other words the displacements field is written as $\mathbf{U}^{\prime}=\mathbf{U}_{\mathbf{S}}+\boldsymbol{\Delta} \mathbf{U}$. The nonlinear forces can then be linearised around the equilibrium position, and from the Taylor expansion it comes:

$$
\mathbf{F}_{\mathbf{n l}}(\mathbf{U})=\mathbf{F}_{\mathbf{n l}}\left(\mathbf{U}_{\mathbf{S}}\right)+\mathbf{J}_{\mathbf{n l}} \Delta \mathbf{U}+\mathcal{O}\left(\Delta \mathbf{U}^{2}\right)
$$

where $\mathbf{J}_{\mathbf{n l}}$ is the Jacobian matrix of the nonlinear efforts around the equilibrium position $\mathbf{U}_{\mathbf{S}}$. It is worth noticing that this Jacobian matrix depends on the system configuration, and so on the status of the internal contacts and on the friction coefficient $\mu$. By injecting Equation 4 in Equation 1, it comes:

$$
\mathbf{M} \Delta \ddot{\mathbf{U}}+\mathbf{C} \Delta \dot{\mathbf{U}}+\left(\mathbf{K}_{n l}+\mathbf{J}_{\mathbf{n l}}\right) \boldsymbol{\Delta} \mathbf{U}=\mathbf{0}
$$




\begin{tabular}{|c|c|c|c|c|c|c|c|c|c|c|}
\hline$\mu$ & 0.01 & 0.15 & 0.25 & 0.35 & 0.45 & 0.55 & 0.65 & 0.75 & 0.85 & 0.95 \\
\hline Ins. $n^{0} 1$ & - & $3878.3 \mathrm{~Hz}$ & $3869.9 \mathrm{~Hz}$ & $3861.9 \mathrm{~Hz}$ & $3856.6 \mathrm{~Hz}$ & $3850.4 \mathrm{~Hz}$ & $1987.8 \mathrm{~Hz}$ & $1979.6 \mathrm{~Hz}$ & $1971.7 \mathrm{~Hz}$ & $1968.2 \mathrm{~Hz}$ \\
\hline Ins. $n^{\circ} 2$ & - & - & $4728.3 \mathrm{~Hz}$ & $4725.3 \mathrm{~Hz}$ & $4721.1 \mathrm{~Hz}$ & $4714.4 \mathrm{~Hz}$ & $3851.4 \mathrm{~Hz}$ & $3854.7 \mathrm{~Hz}$ & $3854.1 \mathrm{~Hz}$ & $3661.4 \mathrm{~Hz}$ \\
\hline Ins. $n^{\circ} 3$ & - & - & - & - & - & $4930.5 \mathrm{~Hz}$ & $4742.5 \mathrm{~Hz}$ & $4785.7 \mathrm{~Hz}$ & $4753.9 \mathrm{~Hz}$ & $3856.8 \mathrm{~Hz}$ \\
\hline Ins. $n^{\circ} 4$ & - & - & - & - & - & - & $4919.3 \mathrm{~Hz}$ & $5384.8 \mathrm{~Hz}$ & $5359.7 \mathrm{~Hz}$ & $4750.3 \mathrm{~Hz}$ \\
\hline Ins. $n^{\circ 5}$ & - & - & - & - & - & - & $4944.1 \mathrm{~Hz}$ & - & $5963.9 \mathrm{~Hz}$ & $5340.2 \mathrm{~Hz}$ \\
\hline Ins. $n^{\circ} 6$ & - & - & - & - & - & - & - & - & - & $5962.8 \mathrm{~Hz}$ \\
\hline
\end{tabular}

Table 2: Unstable frequencies identified with the CEA for a configuration of the brake system for different values of the friction coefficient $\mu$ at the pad/disc interface

The associated eigenvalues problem is defined by:

$$
\left(\lambda^{2} \mathbf{M}+\lambda \mathbf{C}+\left(\mathbf{K}_{n l}+\mathbf{J}_{\mathbf{n l}}\right)\right) \mathbf{\Psi}=\mathbf{0}
$$

where $\lambda$ and $\boldsymbol{\Psi}$ is a pair of complex eigenvalue and eigenvector (i.e. mode shape) of the system. They are determined by solving:

$$
\operatorname{det}\left(\lambda^{2} \mathbf{M}+\lambda \mathbf{C}+\mathbf{K}_{n l}+\mathbf{J}_{\mathbf{n l}}\right)=0
$$

The non-symmetric aspect of the term $\left(\mathbf{K}_{n l}+\mathbf{J}_{\mathbf{n l}}\right)$ makes the eigenvalues complex. The asymptotic stability of the system is determined based on the Lyapunov theory by analysing the sign of the eigenvalues real parts. If all of them are negative, then the system is considered as stable, whereas if at least one of them is positive, then the system is considered as unstable. The real part of the eigenvalue corresponds to the growth ratio of the vibrations associated to the corresponding mode, and the imaginary part corresponds to the angular frequency of the vibrations.

\subsection{Stability of the brake system}

The CEA is performed on the full FEM of the brake system for different values of the friction coefficient $\mu$ at the $\mathrm{pad} /$ disc interface from 0.01 to 0.95 . The unstable frequencies obtained are given Table 2, The system has up to six instabilities between $1960 \mathrm{~Hz}$ and $5960 \mathrm{~Hz}$. The first instability appears between $\mu=0.01$ and $\mu=0.15$ at about $3870 \mathrm{~Hz}$, and the second between $\mu=0.15$ and $\mu=0.25$ at about $4725 \mathrm{~Hz}$. Further simulations demonstrate that it emerges at $\mu=0.23$. In the following, the GMASA method will be applied at $\mu=0.22$ where only one instability is present originally. This test case is chosen at it will be demonstrated through the GMASA that a second one emerges when the modal amplitude of the first mode increases.

\section{Presentation of the GMASA method}

In the following section, the GMASA approach is explained. The general strategy is first described to give the reader a general overview of the approach.

\subsection{General process, main assumptions and workflow}

The GMASA approach has been introduced and validated in detail in [4]. The two main assumptions of this nonlinear approach are:

- the mode shapes and the imaginary part of the eigenvalues remain almost constant when the vibration amplitude is increasing, and so are equal to those computed with the CEA.

- the contribution of a mode on the global non-linear dynamic behaviour (i.e. increasing, decreasing or constant vibration levels) is given by the contribution of the first harmonic of this mode (i.e. contributions of higher harmonics related to a mode are neglected).

From these assumptions, the displacements can be written as:

$$
\boldsymbol{\Delta} \mathbf{U}(t)=\sum_{k=1}^{N_{f}}\left(p_{k}(t) \boldsymbol{\Psi}_{k}^{0} e^{i \omega_{k}^{0} t}+p_{k}(t) \overline{\mathbf{\Psi}_{k}^{0}} e^{-i \omega_{k}^{0} t}\right)
$$

where $N_{f}$ is the number of modes involved in the nonlinear dynamic response, $p_{k}(t)$ is the modal amplitude of the mode $k$ at the instant $t, \mathbf{\Psi}_{k}^{0}$ is the mode shape of the mode $k$ at $p_{k}(0)$ (i.e. obtained from the CEA) and 


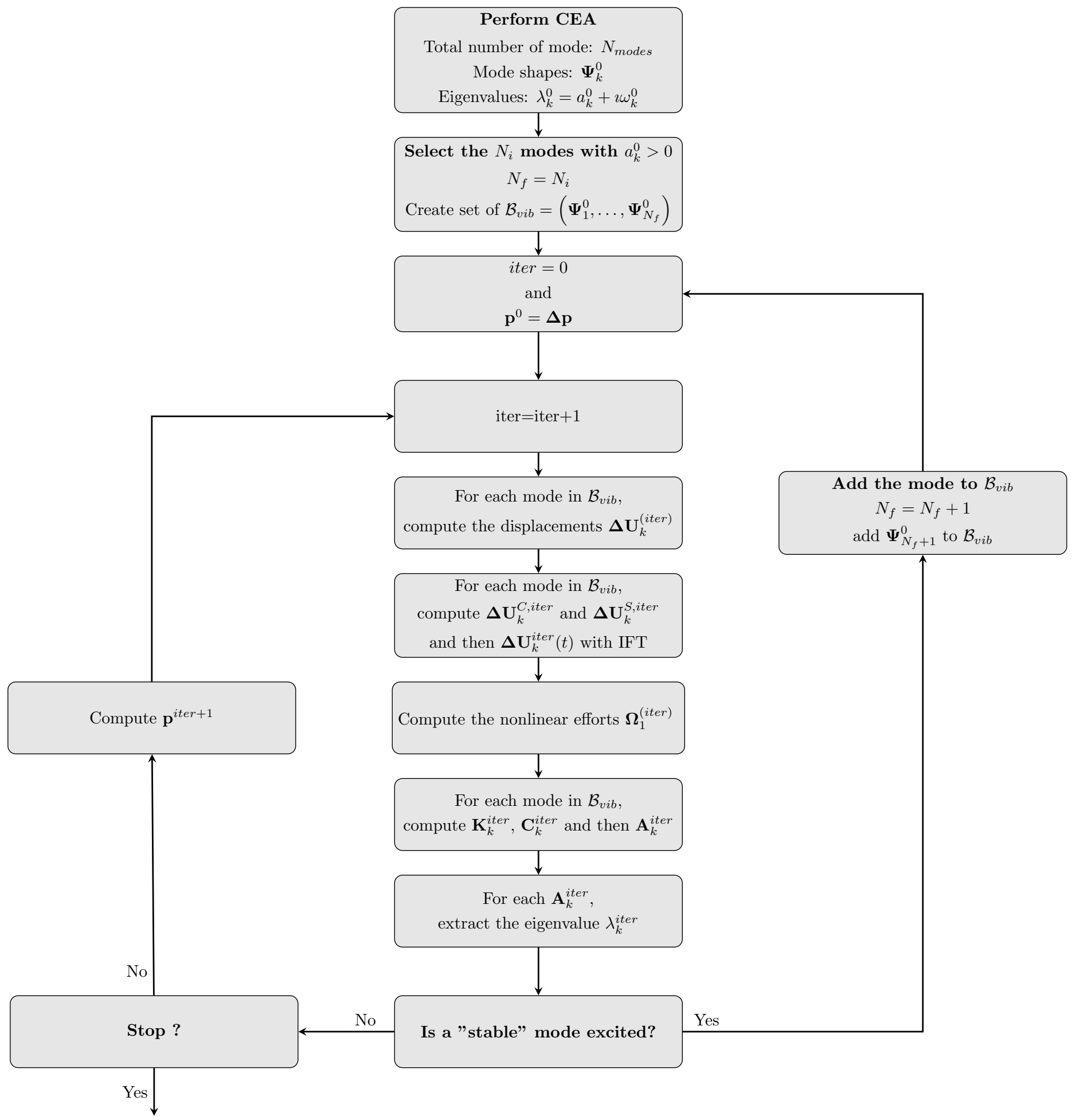

Figure 2: General workflow of the GMASA 
$\omega_{k}^{0}$ is the angular frequency of the mode $k$ at $p_{k}(0)$ (i.e. the imaginary part of the eigenvalue of the mode $k$ computed with the CEA).

From this formulation, the GMASA proposes to identify the $N_{f}$ modes that contribute to the nonlinear dynamic response as well as their modal amplitude $p_{k}$. As a scanning of all the possible $p_{k}$ would represent a too high numerical cost, an iterative process is adopted, where the modal amplitudes are updated at each time step based on the stability behaviour of the system at the previous step. Knowing the modal amplitudes, the displacements are known and the nonlinear efforts are deduced. The system stability can then be studied based on an eigenvalue analysis of the state matrices related to each mode of the system. The main interest of this approach consists in its robustness and its practical implementation that allow its application on large FEM. The general workflow of the GMASA is illustrated in Figure 2, and each step will be detailed in the following.

\subsection{Starting point: the stability analysis - Initialisation of the GMASA}

The first step of the GMASA is the CEA, presented in details in Section 3.1. From this analysis, the mode shapes $\left(\boldsymbol{\Psi}_{k}^{0}\right)$ and the eigenvalues $\lambda_{k}^{0}$ of the structure are computed. The $N_{i}$ unstable modes (i.e. with an eigenvalue with a positive real part) are retained, and without loss of generality are re-numbered from 1 to $N_{i}$. An initial set $\mathcal{B}_{v i b}$ with this $N_{i}$ modes is created. It corresponds to the modes that will be taken into consideration in the GMASA method and more precisely in the equation 8 . It means that at the initialisation of the method, the assumption is made that only the unstable modes identified from the CEA have a contribution in the global non-linear dynamic response. Thus, $N_{f}=N_{i}$. New modes might be added to the set $\mathcal{B}_{\text {vib }}$ (and $N_{f}$ increases), this case will be explained in the following. An initial perturbation $\mathbf{p}_{0}$ of the modal amplitudes is considered [4].

A periodic solution is sought so $\boldsymbol{\Delta} \mathbf{U}$ can be expanded on a generalised Fourier series composed of the angular frequencies of the $N_{f}$ modes, their harmonics and their composition. In the GMASA approach, only the first harmonic of each angular frequency is kept. To mark the difference, the displacements are denoted $\boldsymbol{\Delta} \mathbf{U}_{\mathbf{1 H}}$ and are written:

$$
\boldsymbol{\Delta} \mathbf{U}_{1 \mathbf{H}}=\sum_{k=1}^{N_{f}} \boldsymbol{\Delta} \mathbf{U}_{k}=\sum_{k=1}^{N_{f}} \boldsymbol{\Delta} \mathbf{U}_{k}^{C} \cos \left(\omega_{k}^{0} t\right)+\sum_{k=1}^{N_{f}} \boldsymbol{\Delta} \mathbf{U}_{k}^{S} \sin \left(\omega_{k}^{0} t\right)
$$

where $\boldsymbol{\Delta} \mathbf{U}_{k}^{C}$ and $\boldsymbol{\Delta} \mathbf{U}_{k}^{S}$ are the cosine and sine Fourier coefficients related to the angular frequency $\omega_{k}^{0}$ respectively.

\subsection{Determination of the displacements field}

Given the modal amplitudes $\left(p_{k}^{i t e r}\right)_{k=\left\{1, \ldots, N_{f}\right\}}$ of the modes at iteration iter and Equation 8 , the displacement field at the iteration iter can be written as:

$$
\boldsymbol{\Delta} \mathbf{U}_{\mathbf{1 H}}^{i \text { itr }}(t)=\sum_{k=1}^{N_{f}} \boldsymbol{\Delta} \mathbf{U}_{k}^{i t e r}(t)=\sum_{k=1}^{N_{f}}\left(p_{k}^{i t e r} \mathbf{\Psi}_{k}^{0} e^{i \omega_{k}^{0} t}+p_{k}^{i t e r} \overline{\boldsymbol{\Psi}_{k}^{0}} e^{-i \omega_{k}^{0} t}\right)
$$

The computation of the modal amplitudes at iteration iter is explained later on. The coefficients $\boldsymbol{\Delta} \mathbf{U}_{k}^{C, \text { iter }}$ and $\boldsymbol{\Delta} \mathbf{U}_{k}^{S, \text { iter }}$ are deduced from: $p_{k}^{\text {iter }} \boldsymbol{\Psi}_{k}^{0}=\frac{1}{2}\left(\boldsymbol{\Delta} \mathbf{U}_{k}^{C, \text { iter }}-i \boldsymbol{\Delta} \mathbf{U}_{k}^{S, \text { iter }}\right)$. From the Fourier coefficients, the total displacement field $\boldsymbol{\Delta} \mathbf{U}_{\mathbf{1} \mathbf{H}}^{i \text { ter }}(t)$ is deduced.

\subsection{Computation of the nonlinear efforts}

In the GMASA approach, at each iteration, the modal amplitudes are updated as well as the displacements. From these, the nonlinear efforts related to the contact and friction forces are evaluated. As a first linearisation of the nonlinear forces is done during the CEA, their additional contribution is taken into consideration by considering an additional term $\boldsymbol{\Omega}$ in Equation 5 .

$$
\mathbf{M} \boldsymbol{\Delta} \ddot{\mathbf{U}}+\mathbf{C} \boldsymbol{\Delta} \dot{\mathbf{U}}+\left(\mathbf{K}+\mathbf{J}_{\mathbf{n l}}\right) \boldsymbol{\Delta} \mathbf{U}+\boldsymbol{\Omega}=\mathbf{0}
$$

which is equivalent to:

$$
\mathbf{F}_{\mathbf{n l}}\left(\mathbf{U}_{\mathbf{S}}+\Delta \mathbf{U}\right)=\mathbf{F}_{\mathbf{n l}}\left(\mathbf{U}_{\mathbf{S}}\right)+\mathbf{J}_{\mathbf{n l}} \boldsymbol{\Delta} \mathbf{U}+\boldsymbol{\Omega}
$$

This additional term $\boldsymbol{\Omega}$ corresponds to the error made on the first order Taylor's development. Considering the truncation to the first harmonic and the search for a periodic solution, $\Omega$ is expanded on a generalised Fourier 
series where only first harmonics related to each angular frequency are kept:

$$
\boldsymbol{\Omega}_{\mathbf{1} \mathbf{H}}=\sum_{k=1}^{N_{f}} \boldsymbol{\Omega}_{k}=\sum_{k=1}^{N_{f}} \boldsymbol{\Omega}_{k}^{C} \cos \left(\omega_{k}^{0} t\right)+\sum_{k=1}^{N_{f}} \boldsymbol{\Omega}_{k}^{S} \sin \left(\omega_{k}^{0} t\right)
$$

$\boldsymbol{\Omega}_{\mathbf{1 H}}^{i t e r}$ is deduced from the displacements field $\boldsymbol{\Delta} \mathbf{U}_{\mathbf{1 H}}^{i t e r}$ and from Equation 12 with:

$$
\boldsymbol{\Omega}_{\mathbf{1} \mathbf{H}}^{i t e r}=\sum_{k=1}^{N_{f}} \boldsymbol{\Omega}_{k}^{i t e r}=\mathbf{F}_{\mathbf{n l}}\left(\mathbf{U}_{\mathbf{S}}+\Delta \mathbf{U}_{\mathbf{1}}^{i t e r}\right)-\mathbf{F}_{\mathbf{n l}}\left(\mathbf{U}_{\mathbf{S}}\right)-\mathbf{J}_{\mathbf{n l}} \Delta \mathbf{U}_{\mathbf{1}}^{i t e r}
$$

\subsection{Linearisation of the nonlinear efforts}

The non-linear efforts $\boldsymbol{\Omega}_{\mathbf{1 H}}$ are then linearised with the following approach. Considering the truncation at the first harmonic of $\boldsymbol{\Omega}$ and $\boldsymbol{\Delta} \mathbf{U}$, the Equation 11 is equivalent to:

$$
\mathbf{M} \sum_{k=1}^{N_{f}} \boldsymbol{\Delta} \ddot{\mathbf{U}}_{k}+\mathbf{C} \sum_{k=1}^{N_{f}} \boldsymbol{\Delta} \dot{\mathbf{U}}_{k}+\left(\mathbf{K}+\mathbf{J}_{\mathbf{n l}}\right) \sum_{k=1}^{N_{f}} \boldsymbol{\Delta} \mathbf{U}_{k}+\sum_{k=1}^{N_{f}} \boldsymbol{\Omega}_{k}=\mathbf{0}
$$

From the orthogonality of the Fourier series, it comes:

$$
\forall k \in\left[1, N_{f}\right], \mathbf{M} \boldsymbol{\Delta} \ddot{\mathbf{U}}_{k}+\mathbf{C} \boldsymbol{\Delta} \dot{\mathbf{U}}_{k}+\left(\mathbf{K}+\mathbf{J}_{\mathbf{n l}}\right) \boldsymbol{\Delta} \mathbf{U}_{k}+\mathbf{\Omega}_{k}=\mathbf{0}
$$

The originality of the GMASA method relies in the following linearisation of the nonlinear efforts for each angular frequency $k$ and at each iteration:

$$
\boldsymbol{\Omega}_{k}^{i t e r}=\mathbf{K}_{k}^{i t e r} \boldsymbol{\Delta} \mathbf{U}_{k}^{i t e r}+\mathbf{C}_{k}^{i t e r} \Delta \dot{\mathbf{U}}_{k}^{i t e r}
$$

where the matrices $\mathbf{K}_{k}^{i t e r}$ and $\mathbf{C}_{k}^{i t e r}$ are the equivalent stiffness and damping matrices of the nonlinear efforts on the angular frequency $\omega_{k}^{0}$ at the iteration iter, respectively. These matrices can be obtained analytically. Let $\delta_{k}^{n}$ be the normal displacement of the contact point $n$ projected on the $k$ th angular frequency. For the sake of clarity, the mention to the iteration number is removed here. Because only the first harmonic is considered, it can be written as:

$$
\delta_{k}^{n}(t)=\delta_{c} \cos \left(\omega_{k}^{0} t\right)+\delta_{s} \sin \left(\omega_{k}^{0} t\right)
$$

On the other side, the nonlinear forces at the same contact point $n$ in the direction $d$ (normal or tangential) can be written as:

$$
\boldsymbol{\Omega}_{k}^{n, d}(t)=\alpha_{c} \cos \left(\omega_{k}^{0} t\right)+\alpha_{s} \sin \left(\omega_{k}^{0} t\right)
$$

due to the fact that only the first harmonic is kept. But from Equation 17, it is also equal to:

$$
\mathbf{\Omega}_{k}^{n, d}(t)=\mathbf{K}_{k}^{n, d} \delta_{k}^{n}(t)+\mathbf{C}_{k}^{n, d} \dot{\delta}_{k}^{n}(t)
$$

By arranging Equation 18 and Equation 19 , it comes:

$$
\left\{\begin{array}{lll}
\mathbf{K}_{k}^{n, d}=\frac{\alpha_{c} \delta_{c}+\alpha_{s} \delta_{s}}{\delta_{c}^{2}+\delta_{s}^{2}} & \text { and } \quad \mathbf{C}_{k}^{n, d}=\frac{\alpha_{c} \delta_{s}-\alpha_{s} \delta_{c}}{\omega_{k}^{0}\left(\delta_{c}^{2}+\delta_{s}^{2}\right)} & \text { if } \delta_{c} \neq 0 \text { or } \delta_{s} \neq 0 \\
\mathbf{K}_{k}^{n, d}=0 & \text { and } \quad \mathbf{C}_{k}^{n, d}=0 & \text { otherwise }
\end{array}\right.
$$

It is worth emphasizing here that the $\left(\delta_{c}\right)$ and the $\left(\delta_{s}\right)$ are known from Equation 10 and the $\left(\alpha_{c}\right)$ and the $\left(\alpha_{s}\right)$ from Equation 14 (and by applying a Fourier transform).

\subsection{Contribution of each mode in the nonlinear dynamic response}

Coupling the Equation 15 and the Equation 17 it comes for each angular frequency $\omega_{k}^{0}$ and at each iteration iter:

$$
\forall k \in\left[1, N_{f}\right], \mathbf{M} \boldsymbol{\Delta} \ddot{\mathbf{U}}_{k}^{i t e r}+\left(\mathbf{C}+\mathbf{C}_{k}^{i t e r}\right) \boldsymbol{\Delta} \dot{\mathbf{U}}_{k}^{i t e r}+\left(\mathbf{K}+\mathbf{J}_{\mathbf{n l}}+\mathbf{K}_{k}^{i t e r}\right) \boldsymbol{\Delta} \mathbf{U}_{k}^{i t e r}=\mathbf{0}
$$

Written in the state-space form, it is equivalent to:

$$
\forall k \in\left[1, N_{f}\right], \dot{\mathbf{Y}}_{k}^{i t e r}=\mathbf{A}_{k}^{i t e r} \mathbf{Y}_{k}^{i t e r}
$$


where $\mathbf{Y}_{k}^{i t e r}=\left[\boldsymbol{\Delta} \mathbf{U}_{k}^{i t e r} \boldsymbol{\Delta} \dot{\mathbf{U}}_{k}^{i t e r}\right]^{T}$, and where each matrix $\mathbf{A}_{k}^{i t e r}$ is equal to:

$$
\mathbf{A}_{k}^{i t e r}=\left[\begin{array}{cc}
\mathbf{0} & \mathbf{I} \\
-\mathbf{M}^{-1}\left(\mathbf{K}+\mathbf{J}_{\mathbf{n l}}+\mathbf{K}_{k}^{i t e r}\right) & -\mathbf{M}^{-1}\left(\mathbf{C}+\mathbf{C}_{k}^{\text {iter }}\right)
\end{array}\right]
$$

Hence, each mode $k$ considered in the GMASA method is characterised by its own subset of equation, called sub-system in the following, and its dynamic matrix $\mathbf{A}_{k}^{i t e r}$ at the iteration iter.

From this formulation, one can analyse the temporal evolution of the dynamic solution linearised around an equilibrium position for a given modal amplitude, in other words it is possible to know if the vibrations amplitude of the mechanical system are increasing, decreasing or remain constant. This analysis is done by analysing the eigenvalue related to the mode $k$ of the matrix $\mathbf{A}_{k}$ for each mode $k$. Indeed, each $\mathbf{Y}_{k}$ is only related to $\omega_{k}$ and there is no other contribution from other angular frequencies, this comes from the orthogonality of the Fourier series. Thus, the contribution of the mode $k$ in the dynamic response is directly assessed through the sign of the real part of its eigenvalue $\lambda_{k}$ from the matrix $\mathbf{A}_{k}$. As the contribution of the mode $k$ in the solution $\boldsymbol{\Delta} \mathbf{U}_{\mathbf{1 H}}$ is given by:

$$
\boldsymbol{\Delta} \mathbf{U}_{k}(t)=p_{k}^{i t e r}\left(\boldsymbol{\Psi}_{k}^{0} e^{e_{k}^{i t e r} t}+\overline{\Psi_{k}^{0}} e^{\overline{\lambda_{k}^{i t e r}} t}\right)
$$

if the real part of $\lambda_{k}^{i t e r}$ is positive, then the associated vibration amplitudes increase, if the real part is negative, then the associated vibration amplitudes decrease and if the real part is null then the vibration amplitudes remain constant.

If in a sub-system $k$, a mode $j$ with $j \neq k$ has a positive real part, then it is necessary to consider the sub-system associated to the mode $j$ to know if this divergence is effectively translated by a divergence in the dynamic solution. If this mode $j$ is identified as stable by the CEA (i.e. $\operatorname{Real}\left(\lambda_{j}^{i \text { ter }=0}\right)=0$ ), then it is necessary to add this mode in the GMASA modal basis $\mathcal{B}_{\text {vib }}$.

\subsection{Determination of the modal amplitude}

At each iteration, the vector of modal amplitude $\mathbf{p}^{i t e r}$ is updated. This is done by coming back to a physical and temporal description of the vibratory response. If a mode $k$ is characterised by a modal amplitude $p_{k}^{i t e r}\left(t_{i t e r}\right)$ at the iteration iter (that can be seen as an instant $t_{i t e r}$ ), and by an eigenvalue $\lambda_{k}^{i \text { iter }}=a_{k}^{i \text { iter }}+\imath \omega_{k}^{i \text { iter }}$, then the modal amplitude at the instant $t_{i t e r+1}=t_{i t e r}+\delta t$ is linked to $p_{k}^{i t e r}\left(t_{i t e r}\right)$ and $\lambda_{k}^{i t e r}$ by

$$
p_{k}^{i t e r+1}=p_{k}^{i t e r} \exp \left(a_{k}^{i t e r} \delta t\right)
$$

Two parameters must be chosen carefully in the GMASA method as shown in detail in [47, namely the initialisation $\boldsymbol{\Delta} \mathbf{p}$ and the time step $\delta t$. The initialisation is a perturbation and should not be "too large", but a too small perturbation would lead to a larger number of iteration to reach the stationary response. Considering the choice of $\delta t$, it must be chosen so that the approximation given by Equation 26 remains valid.

The method is stopped when a stop criterion is reached. Depending on the choice of the user, it can be a maximum of iteration, a maximum time or when the modal amplitudes are not evolving anymore.

\section{Nonlinear analysis of an industrial automotive brake system with the GMASA}

\subsection{Mono-instability case}

In this section, the GMASA is used to predict the nonlinear dynamic behaviour of the brake system. As a reminder, the friction coefficient at the pad/disc interface is fixed at $\mu=0.22$, where one unstable mode is identified with the CEA at $3870 \mathrm{~Hz}$ with a real part equal to 87. This mode is denoted mode $A$ in the following and its mode shape is represented in Figure $4(\mathrm{a}, \mathrm{b})$. This mode is the combination of a disc mode and of a bracket mode. The maximum of vibrations is located at one side of the caliper. As there is a unique unstable mode identified by the CEA, the MASA method is applied [46. It consists in the GMASA with a unique unstable mode, so a scanning on the modal amplitude can be performed directly. The evolution of the real parts and of the frequencies from the successive $\mathbf{A}_{\text {modeA }}^{\text {iter }}$ are given in Figure 3 . The eigenvalues are followed by tracking the associated mode shapes with a MAC criterion. For the sake of clarity in Figure 3(b), only the frequencies of modes where the real part of their eigenvalues is non-zero at some value of the modal amplitude are displayed. It is worth reminding here that the eigenvalues are the eigenvalues obtained in the subsystem associated to the mode A for each level of its modal amplitude.

In Figure 3(a), the real part of the eigenvalues for different modal amplitudes of the mode A are given. Depending on the modal amplitude of the mode A, five different stages are observed: 


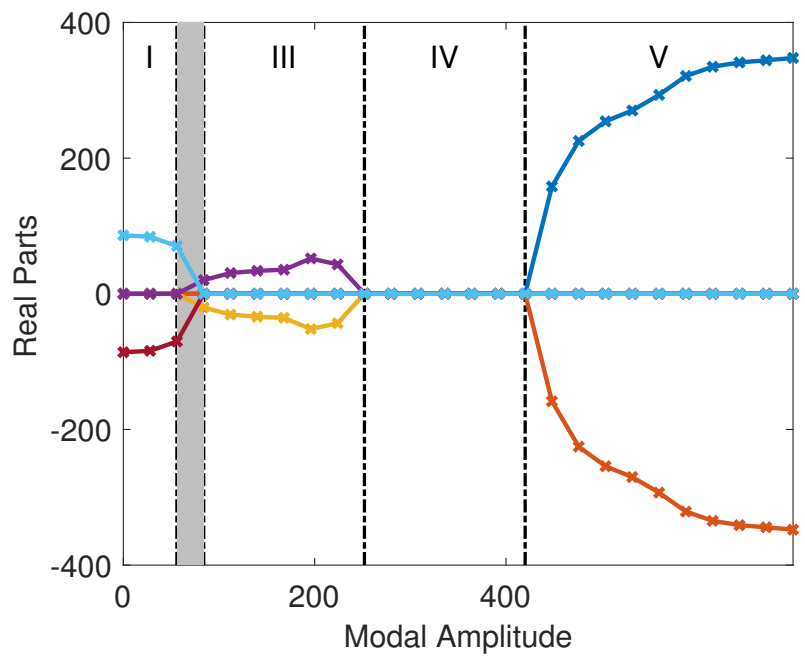

(a)

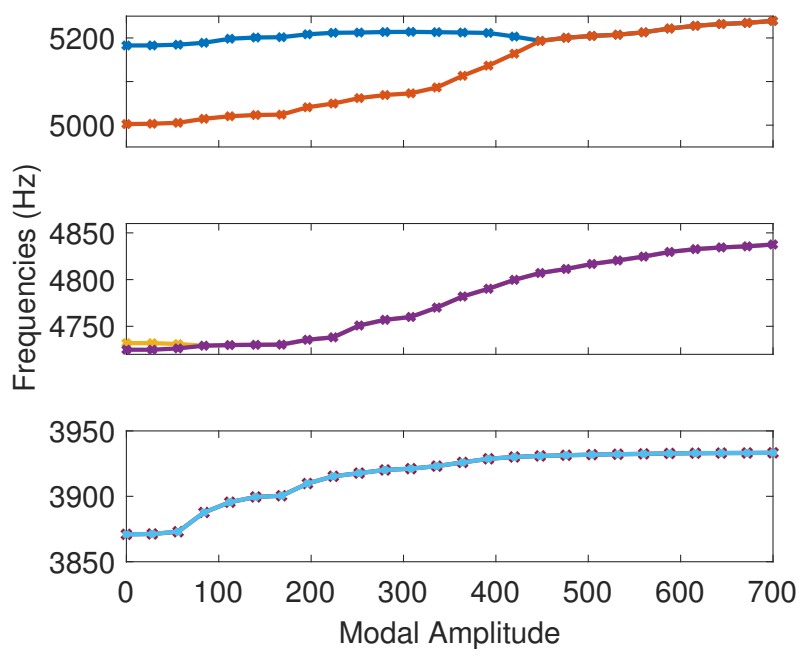

(b)

Figure 3: Evolution of the real parts (left) and of the frequency (right) for different level of modal amplitude of the unstable mode at $3870 \mathrm{~Hz}$

- Stage I: the mode A has an eigenvalue with a positive real part which decreases when increasing the modal amplitude. So, the levels of vibrations of the brake system increase, but less and less quickly as the real part decreases, and the system dynamic is driven by a unique mode.

- Stage II: the mode A has still a positive real part, but a new mode at $4750 \mathrm{~Hz}$, denoted mode $B$, also exhibits an eigenvalue with a positive real part. This mode B is a new potential instability, and to conclude on its impact on the global dynamic behaviour of the brake system, a multi-instability GMASA analysis must be carried on. This new potential instability comes from a mode coupling between two modes with frequencies equal to $4725 \mathrm{~Hz}$ and $4732 \mathrm{~Hz}$. This mode coupling comes from the increase in the modal amplitude of mode A.

- Stage III: the real part of the mode $\mathrm{A}$ is equal to 0 , which means the contribution of the mode $\mathrm{A}$ in the system dynamic is constant. Only the mode B has a positive real part, and to conclude on its consequence on the system stability a multi-instability GMASA analysis is required.

- Stage IV: all the real parts are null; the system experiences self-sustained vibrations and its dynamic is characterized by a limit cycle.

- Stage V: a new potential unstable mode appears at $5200 \mathrm{~Hz}$, denoted mode $C$, with a positive real part. This potential instability comes from a mode coupling between two modes at $5002 \mathrm{~Hz}$ and $5183 \mathrm{~Hz}$. To get its influence in the system dynamic, a multi-instability GMASA analysis must be performed.

Considering a small perturbation of the nonlinear static equilibrium position, the modal amplitude of the mode A would be very low, and the levels of vibration would increase as the real part of the eigenvalue of the mode A is positive (see stage I). These vibration levels would increase, but less and less quickly as the real part decreases for increasing modal amplitude. However, before the system reaches a steady-state solution (i.e. a null real part), the mode B has an eigenvalue with a positive real part. This mode is a potential instability, and according to the GMASA approach, to get its contribution on the increase or on the decrease of the vibratory levels, it must be added in a multi-instability GMASA analysis. The mode $\mathrm{C}$ is not considered here as it appears for high level of modal amplitude for the mode A, after a stage where the latter has stabilized (i.e. a null real part in stage IV). Hence, the system would reach this stage IV where the vibratory levels remain constant and so higher modal amplitude would never be reached. For this reason, in the following part, a multi-instability GMASA analysis is performed where two modes are considered: the mode $\mathrm{A}$ at $3870 \mathrm{~Hz}$ and the mode $\mathrm{B}$ at $4750 \mathrm{~Hz}$. The mode shapes of these two modes are given Figure 4. The mode A at $3870 \mathrm{~Hz}$ is the combination of a disc mode and of a bracket mode, where the maximum of vibration is observed at one side of the caliper. The mode $\mathrm{B}$ at $4750 \mathrm{~Hz}$ involves mostly the outer pad as well as the back of the bracket, and the maximum of vibrations is observed on the back of the bracket. 


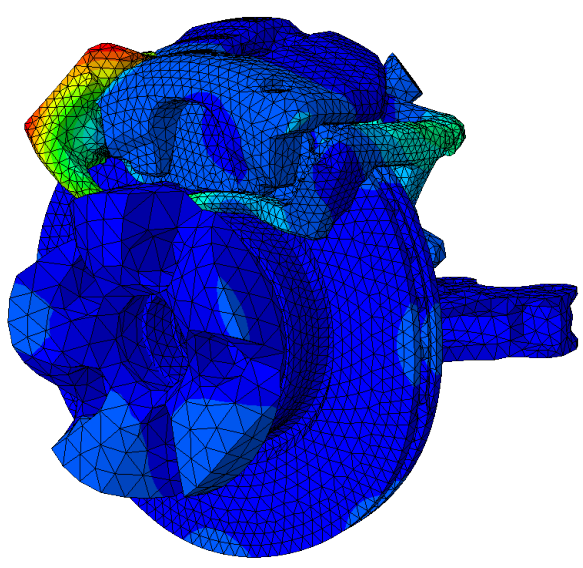

(a) Mode A - $3870 \mathrm{~Hz}-$ View 1

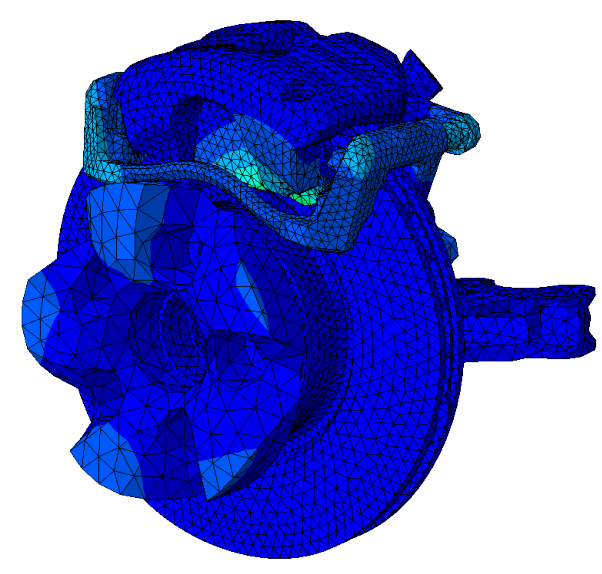

(c) Mode B - $4750 \mathrm{~Hz}-$ View 1

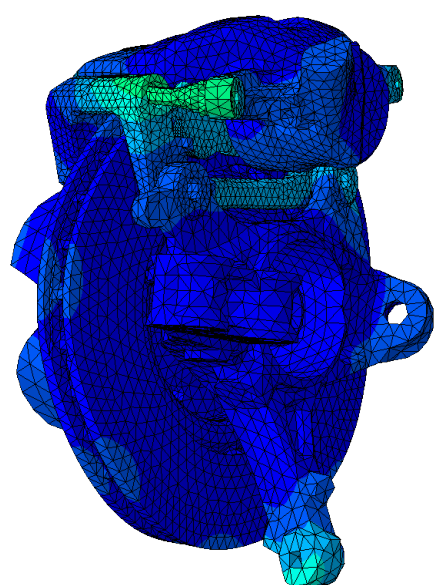

(b) Mode A - $3870 \mathrm{~Hz}$ - View 2

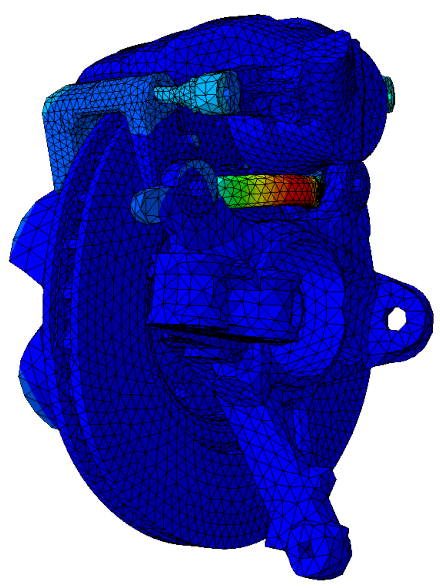

(d) Mode B - $4750 \mathrm{~Hz}$ - View 2

Figure 4: Mode shapes of the modes considered in the GMASA analysis

\subsection{Multi-instability case}

A second analysis is then carried out with the GMASA method to take into consideration this second mode. The method is initialised with two modes: the unstable mode identifies by the CEA at $3870 \mathrm{~Hz}$, and the mode potentially unstable identified by the MASA at $4750 \mathrm{~Hz}$. Low modal amplitudes are assigned to both modes for the initialisation, namely $p_{1}^{0}=p_{2}^{0}=2$. The evolution of the modal amplitudes, of the real part of the eigenvalues and of the frequencies of each considered mode are given in Figure 5 . They correspond to the evolution of the parameters that characterise each considered mode (i.e. the real part of the eigenvalue and the frequency of the mode A (resp. mode B) in the subsystem A (resp. B)). In Figure 6, the evolution of the eigenvalues in each subsystem are given.

From Figure 5, one can observe that initially only the mode A is unstable with an eigenvalue with a real part equal to 87 and with a frequency of $3871 \mathrm{~Hz}$. Until $t_{M A S A}=0.045 \mathrm{~s}$, its real part remains almost constant and its modal amplitude increases, and so the total dynamic response is divergent. Then, from $t_{M A S A}=0.045 \mathrm{~s}$, the mode $\mathrm{B}$ has the real part of its eigenvalue that starts to increase and becomes positive, whereas the mode $\mathrm{A}$ has the real part of its eigenvalue that decreases quickly and becomes null. From $t_{M A S A}=0.05 \mathrm{~s}$, the mode $\mathrm{A}$ has an eigenvalue with a zero-real part and is stabilised with a modal amplitude equal to about 65 . The mode $\mathrm{B}$ has a real part that remains constant and equal to 21 from $t_{M A S A}=0.05 \mathrm{~s}$ to $t_{M A S A}=0.17 \mathrm{~s}$, and its modal amplitude increases. From $t_{M A S A}=0.17 \mathrm{~s}$, the real part of the eigenvalue of the mode B increases again and more strongly from $t_{M A S A}=0.21 \mathrm{~s}$. The modal amplitude of this mode increases quickly during this period. Finally, at $t_{M A S A}=0.22 \mathrm{~s}$, the real part of the eigenvalue of the mode B decreases quickly and becomes null at $t_{M A S A}=0.23 \mathrm{~s}$. Its modal amplitude is then stabilised at $p=195$.

The evolution of the eigenvalues of the two sub-systems given in Figure 6 demonstrates that no other potential instability emerges in one of the sub-system, and so it is not necessary to consider more modes in the 


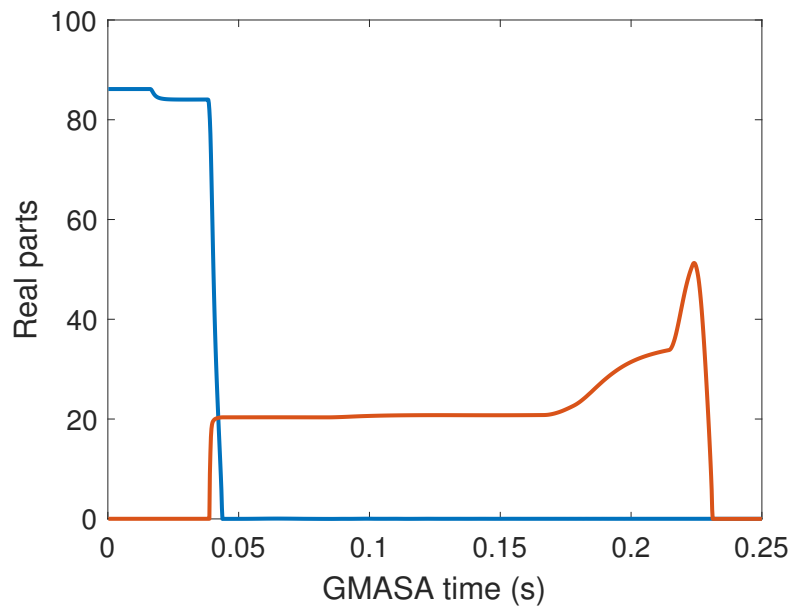

(a)

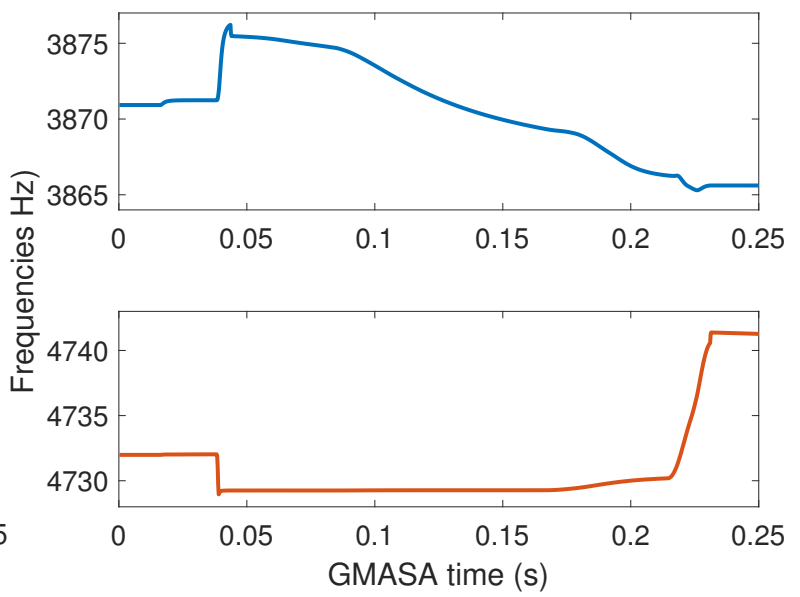

(b)

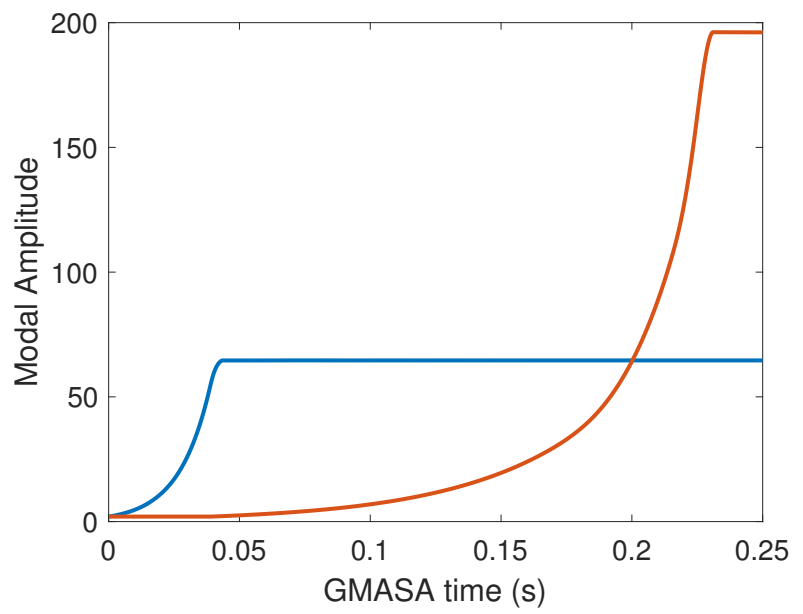

(c)

Figure 5: Evolution of the modal parameters of mode A (blue) and mode B (orange): real parts of the eigenvalue (a), frequencies (b) and modal amplitudes (c)

GMASA method. To summarize, the GMASA method indicates the following contributions:

- $t_{\text {MASA }}<0.045 \mathrm{~s}$ : a unique mode with a frequency of $3870 \mathrm{~Hz}$ brings an amplitude divergence in the vibratory response of the brake system,

- $0.045 s<t_{\text {MASA }}<0.05 s$ : two modes of frequencies $3870 \mathrm{~Hz}$ and $4730 \mathrm{~Hz}$ bring a divergence in amplitude of the solution,

- $0.05 s<t_{M A S A}<0.23 \mathrm{~s}$ : one mode with a frequency of $3870 \mathrm{~Hz}$ brings a constant contribution to the vibratory response, a second mode at $4730 \mathrm{~Hz}$ brings a divergence in the dynamic solution,

- $t_{\text {MASA }}>0.23 \mathrm{~s}$ : two modes at $3870 \mathrm{~Hz}$ and $4730 \mathrm{~Hz}$ with a constant contribution to the vibratory response characterise an approximated quasi-periodic steady-state solution.

As a conclusion, the structure has a dynamic response driven by two modes at $3870 \mathrm{~Hz}$ and $4730 \mathrm{~Hz}$ with modal amplitudes equal to 65 and 195, respectively. The mode shapes of these two modes are given in Figure 4 This result demonstrates the interest of using the GMASA method as a complement of the CEA. Indeed, only one unstable mode was predicted by the CEA computation, whereas two are present in the dynamic response of the brake system, and the modal amplitude of this second mode is much higher than the contribution of the mode identifies by the CEA. Even if this mode was identified as unstable by the CEA for higher friction coefficients (see Table 2), the GMASA allows for a more precise identification of the stability limit of each mode. It is worth highlighting here that the emergence of the second is due to the coupling created by the nonlinear forces between the two modes due to Equation 14 . 


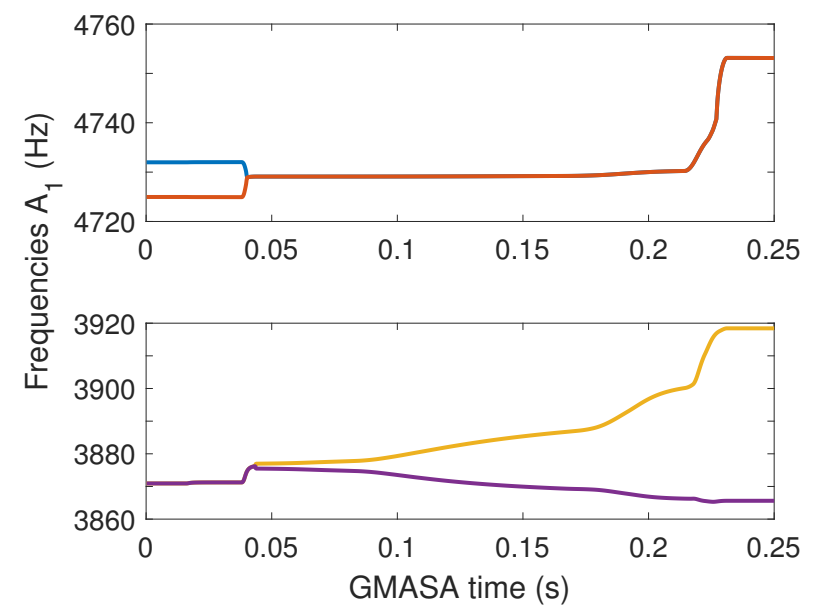

(a) Mode A

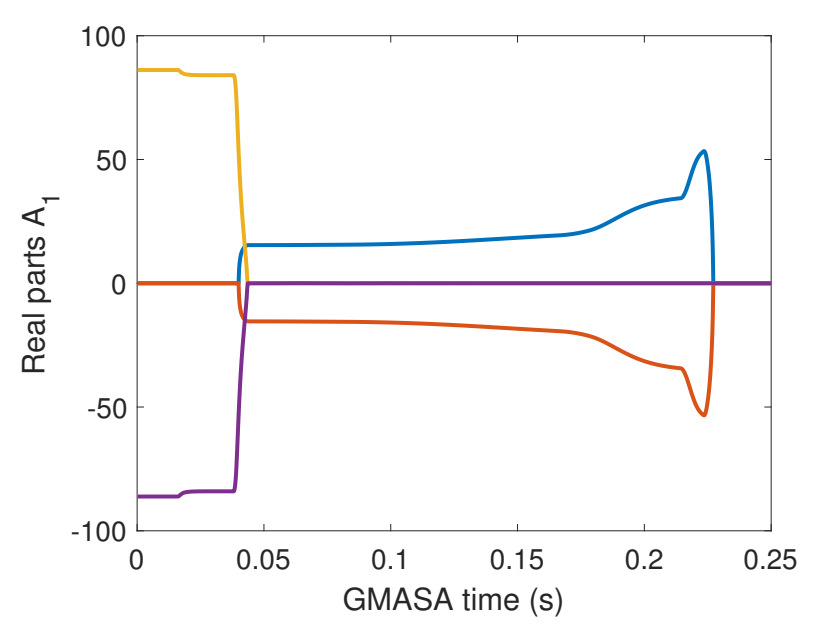

(c) Mode A

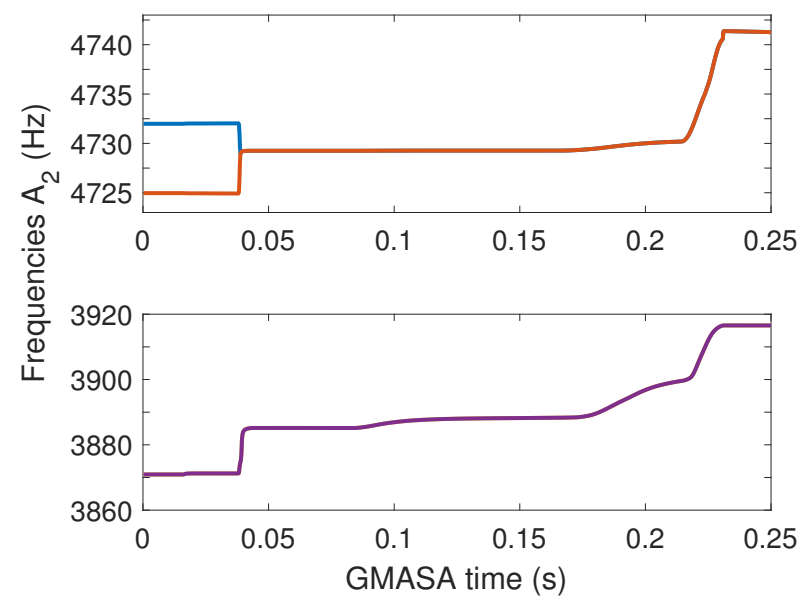

(b) Mode B

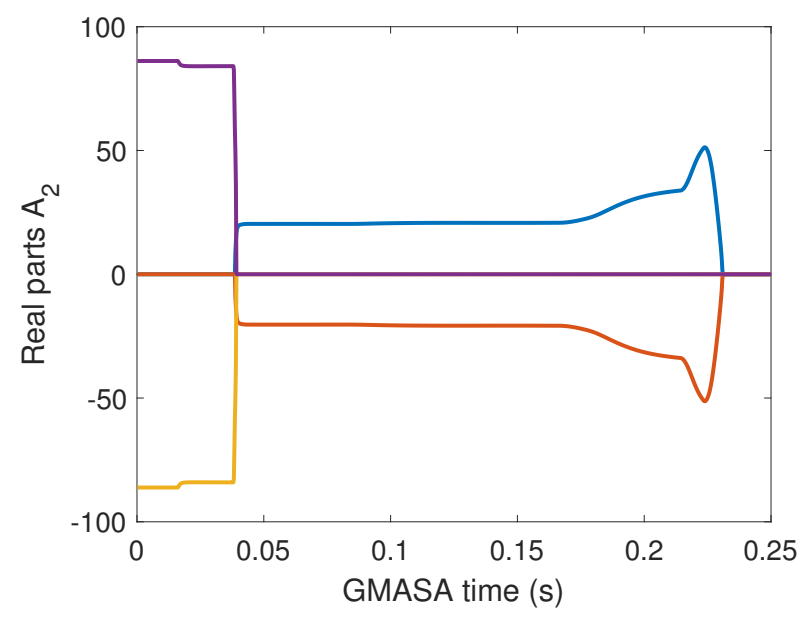

(d) Mode B

Figure 6: Evolution of the frequencies $(\mathrm{a}, \mathrm{b})$ and the real parts $(\mathrm{c}, \mathrm{d})$ in the sub-system of the mode $\mathrm{A}(\mathrm{a}, \mathrm{c})$ and of the mode $\mathrm{B}(\mathrm{b}, \mathrm{d})$

\subsection{Analysis of the quasi-periodic solution}

Moreover, one of the main interest of the GMASA method compared to the CEA is that the vibration levels are approximated. Indeed, the quasi-periodic steady-state dynamic response of the system is approximated by:

$$
\boldsymbol{\Delta} \mathbf{U}_{\mathbf{1}}(t)=\mathbf{U}_{\mathbf{S}}+p_{1} \mathbf{\Psi}_{1}^{0} e^{i \omega_{1}^{0} t}+p_{1} \overline{\Psi_{1}^{0}} e^{-i \omega_{1}^{0} t}+p_{2} \boldsymbol{\Psi}_{2}^{0} e^{i \omega_{2}^{0} t}+p_{2} \overline{\mathbf{\Psi}_{2}^{0}} e^{-i \omega_{2}^{0} t}
$$

with $p_{1}=65$ and $p_{2}=195, \omega_{1}^{0}=2.4310^{4} \mathrm{rad} / \mathrm{s}$ and $\omega_{2}^{0}=2.9710^{4} \mathrm{rad} / \mathrm{s}$, and where $\boldsymbol{\Psi}_{1}^{0}$ and $\boldsymbol{\Psi}_{2}^{0}$ are the mode shapes of the two modes obtained from the CEA computation.

The contact status at the pad/disc interfaces for the steady-state response are given in Figure 7. As a reminder, the contact interfaces are reduced to 212 points in total. The left pad corresponds to the outer pad, and the right pad to the inner pad. One can notice that the contact status distribution is different from one pad to another. Indeed, the inner pad has more points that are always in contact (see the different number of red points), and also have more points that experience contact and separation (see the green points). For both pads, the points which are furthest from the rotation center are always in contact.

For a deeper insight in the brake dynamic, the evolution of these contact conditions at the pad/disc interfaces over the GMASA time is given in Figure 7 for each pad. At the beginning, the contacts are either always in contact or always separated. Then, when the modal amplitudes increase, more and more contact points start to experience impact. These micro-impacts during the vibrations are at the origin of the stabilisation of the modal response. One can also observe that the evolution of the contact conditions for the inner pad (top figure) are much more complex than for the outer pad, where only a limited number of contact points experience a 


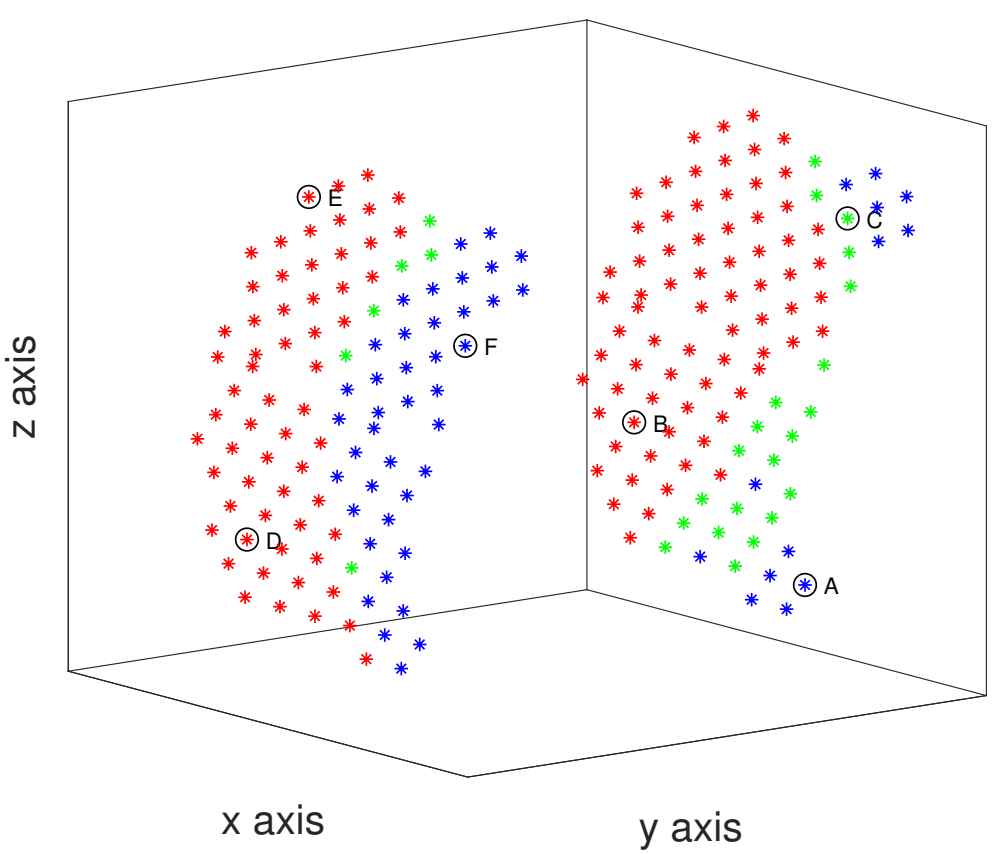

Figure 7: Contact status at the steady-state response at the pads/disc interfaces: always separated (blue), always in contact (red), contact and separation (green)

modification of their contact conditions when the modal amplitudes vary. For the outer pad (bottom figure), the changing in the contact conditions start later (after $0.18 \mathrm{~s}$ of the GMASA time), when the modal amplitude of the second mode increases drastically. For the inner pad (top figure), impact starts much earlier from about $0.02 \mathrm{~s}$. Around about $0.07 \mathrm{~s}$, the contact statuses remain constant, as well as the modal amplitude of the mode at $3870 \mathrm{~Hz}$, until about $0.14 \mathrm{~s}$, when the contribution of the mode at $4730 \mathrm{~Hz}$ becomes relatively important. Finally, when the modal amplitude of the mode at $4730 \mathrm{~Hz}$ increases, more and more contact points experience impact until a stabilisation of the modal amplitude of this second mode is reached. Finally, impacts coming from the vibration of the mode at $3870 \mathrm{~Hz}$ are not enough to stabilise the vibrations, so the vibration amplitude keeps increasing until the modal amplitude of the mode at $4730 \mathrm{~Hz}$ reaches high enough levels so more points experience also impact leading to a stabilisation of the dynamic response to a torus motion. A torus motion can be seen as an extension of the limit cycle notion, as a quasi-periodic motion is composed of incommensurate frequencies and is described in dimension 2. To be noted that the stabilisation of the final torus motion is more exactly due to the nonlinear interactions of both the contact/no-contact states and the cubic non-linear contribution at the frictional contact interface.

Additionally, the quasi-periodic orbits obtained for some contact points are given in Figure 9 for the inner pad, and Figure 10 for the outer pad. The quasi-periodic orbits are given for the points $\mathrm{A}$ to $\mathrm{F}$ displayed in Figure 7, in the three directions, the normal of the contact being the y-axis. The orbits of the disc (resp. pads) points are in blue (resp. orange). The quasi-periodic orbits differ from one point to another and have more or less complex shape, and the following observations can be done:

- separation case - points A and F: the orbits amplitude in the normal direction (i.e. $y$ direction) of the pad is larger than the one on the disc (see the orange orbits larger than the blue one). Larger displacements are observed for the outer pad than for the inner one (about $1.8 \mathrm{E}-3 \mathrm{~m}$ versus $4 \mathrm{E}-5 \mathrm{~m}$ ) for the pad, but displacements are of the same order of magnitude for the disc in this direction (around 1e-5 m). Tangential behaviours are however different as for the outer pad (point F) disc displacements are larger in the $x$ direction (tangent to the rotational speed) whereas for the point A, pad displacements are much larger in the $x$ direction. In the $z$ direction, for both cases, the displacements are larger for the pad than for the disc.

- contact case - points B, D and E: in the $y$ direction (normal of the contact) the displacement amplitude of the disc is always larger than the amplitude of the pad. For the two other directions, in the contact plane, the displacements of the pad and the disc are of similar amplitude for the outer pad (points D and E), whereas for the inner pad (point B), the pad displacement amplitude is much larger than for the disc in the $x$ direction. For B and D, the largest vibration amplitudes are observed in the $y$ direction (normal direction), whereas for the point $\mathrm{E}$, the largest displacement are observed in the $z$ direction (radial 

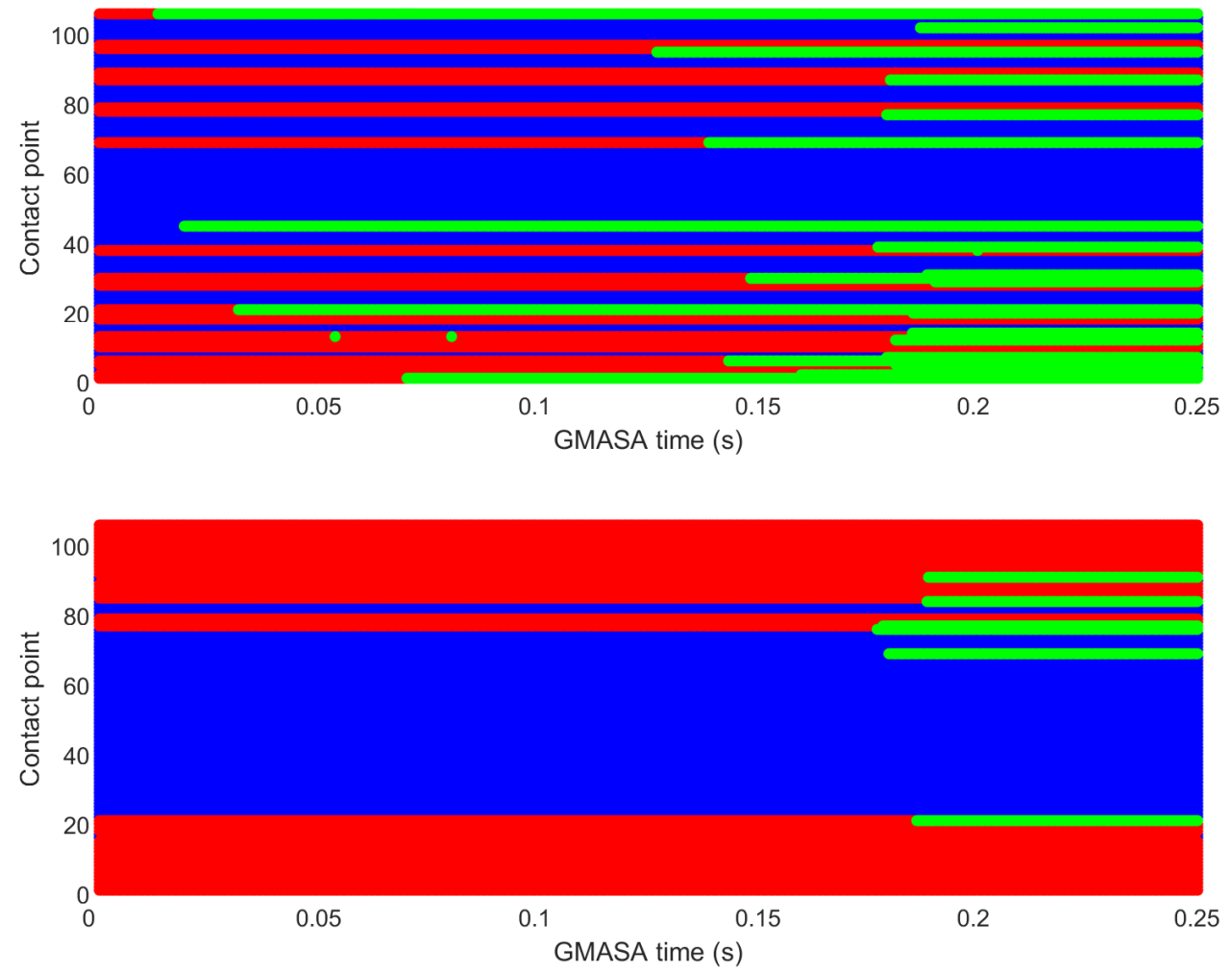

Figure 8: Evolution of the contact status for the inner pad (top) and the outer pad (bottom): blue = always separate, red $=$ always contact, green $=$ contact $/$ sep

direction).

- impact case - point $\mathrm{C}$ : the pad vibration amplitudes are always smaller in all direction and the largest amplitudes are observed in the $z$ direction (radial direction).

Finally, the evolution of the quasi-periodic orbits over the GMASA time are given in Figure 11 for the points $\mathrm{C}$ and $\mathrm{G}$ and for the points $\mathrm{D}$ and $\mathrm{F}$ in Figure 12 . The first figure corresponds to points that experience impact, whereas in Figure 12 the point is always in contact and $\mathrm{F}$ is always open. The quasi-periodic orbits are displayed every $0.03 \mathrm{~s}$ for the sake of readability. As a reminder, the direction normal to the contact is the $y$ axis. At $0 \mathrm{~s}$, all the quasi-periodic orbits have a small amplitude, which corresponds to the initial perturbation. Then, the different amplitudes are growing up over the GMASA time. From a global point of view, the growing of the quasi-periodic orbits has similar behaviour where a plateau is observed between $0.06 \mathrm{~s}$ and $0.18 \mathrm{~s}$ before the amplitudes start growing exponentially. This has to be compared to the Figure 5(c) where during this time interval, the modal amplitude of the first mode remains constant, and the modal amplitude of the second mode increases slowly, which is translated by a slow evolution of the quasi-periodic orbits. After $0.18 \mathrm{~s}$, the modal amplitude of the second mode increases exponentially and the quasi-periodic orbits are also following this evolution. To go into the details:

- in the normal direction (i.e. $y$ axis), the behaviour between the four points is clearly different. Indeed, at the beginning, the amplitudes of the disc and the pad are similar for the four points. For the point $\mathrm{C}$, the vibration amplitude of the pad remains somewhat constant whereas the vibration amplitudes of the disc are increasing. The opposite behaviour is observed for the point G, i.e. an increase of the vibration for the pad and less increase for the disc. For the point $\mathrm{F}$ a drastic increase of the pad vibration is observed and the disc vibrations remain negligible at this point. For the point $\mathrm{D}$, they remain somewhat similar during all the time evolution. Considering the shape of the orbits, in all cases they are circle at the beginning, illustrating the presence of only one fundamental frequency, and have complex shape at the end, illustrating the presence of the second mode in the dynamic response.

- in the $x$ direction, for the points $\mathrm{C}, \mathrm{D}$ and $\mathrm{F}$, the disc vibration amplitude is much larger than the pad 


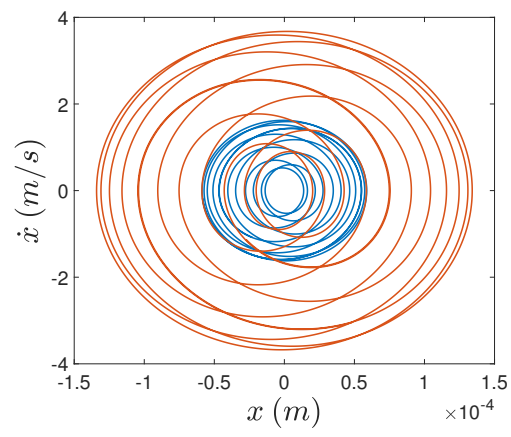

(a) Point A - x axis

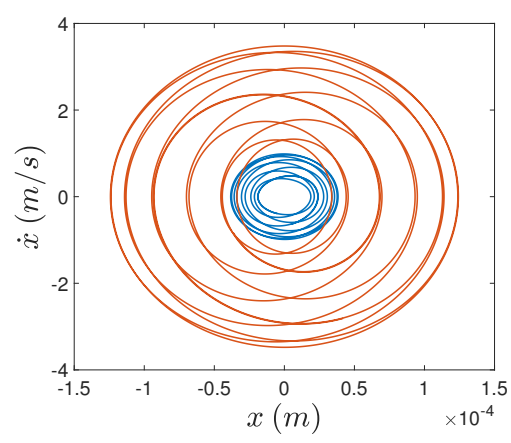

(d) Point B - x axis

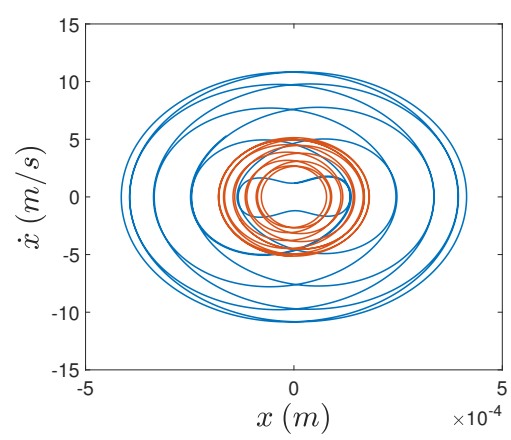

(g) Point $\mathrm{C}-\mathrm{x}$ axis

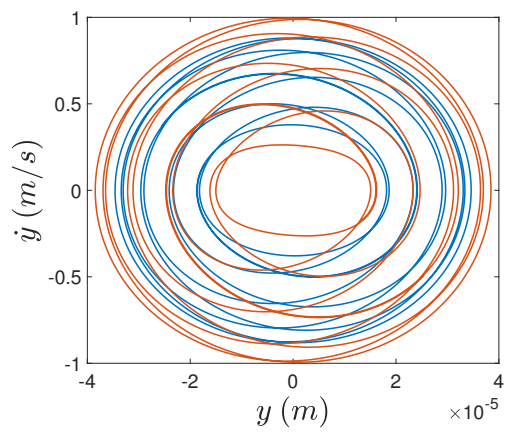

(b) Point A - y axis

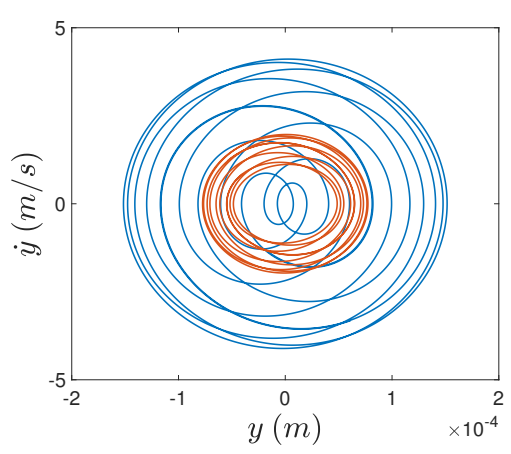

(e) Point B - y axis

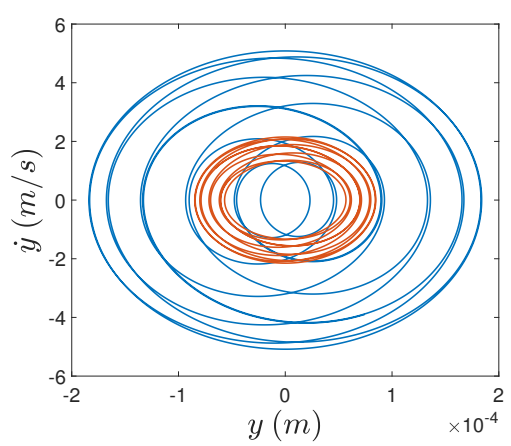

(h) Point $\mathrm{C}-\mathrm{y}$ axis

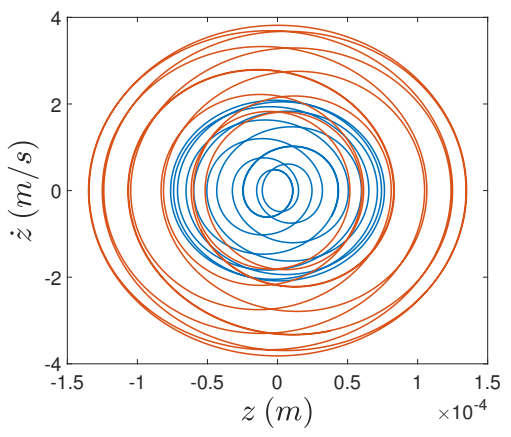

(c) Point $\mathrm{A}-\mathrm{z}$ axis

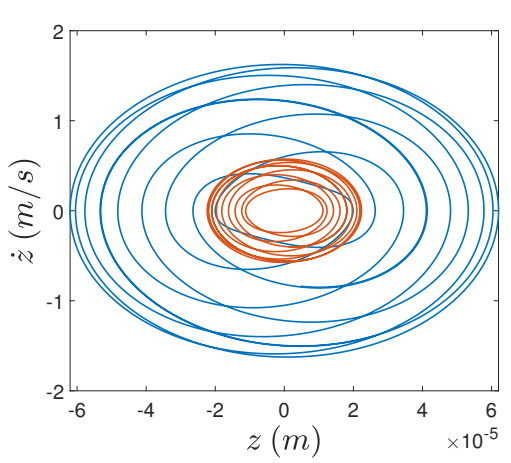

(f) Point B - z axis

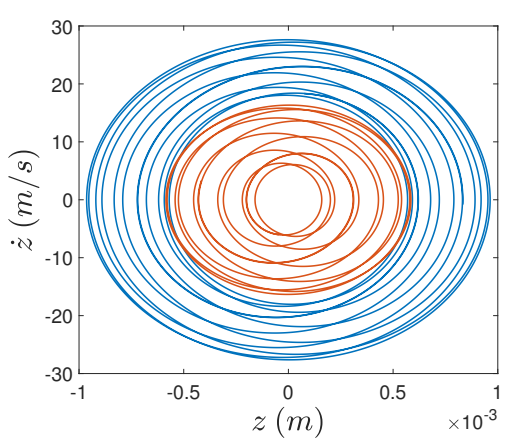

(i) Point $\mathrm{C}-\mathrm{z}$ axis

Figure 9: Disc torus motions (blue) and pad torus motions (orange) of the points A, B and C of the inner pad

vibration amplitude. On the opposite, for the point $\mathrm{G}$, only the pad vibrations are increasing. At the beginning, the orbits are circle and at the end they present a complex shape, showing again the presence of the second mode in the dynamic response. The quasi-periodic orbits shape of the disc is more complex than the pad ones for the points C, D and F, whereas a more complex orbit is observed for the pad at the point $\mathrm{G}$. This result is only the consequence of the distribution of the contributions of the two unstable modes on the nodes of observation.

- in the $z$ direction, very different behaviours are observed. Indeed, for the point $\mathrm{C}$ the amplitude of the disc and the pad are of similar amplitude at the beginning, whereas for points $\mathrm{G}$ and $\mathrm{F}$ larger amplitudes are observed on the disc and for the point $\mathrm{D}$ the largest amplitudes are observed on the pad. For the point $\mathrm{D}$, the pad keeps a larger vibration amplitude over the GMASA time and the quasi-periodic orbits of both the pad and the disc are becoming more and more complex. Whereas for the points $\mathrm{G}$ and $\mathrm{F}$, the vibration amplitudes of the pad are becoming larger and larger and finally represent the largest amplitude at the end. For the point $\mathrm{C}$, the disc vibration amplitudes become larger than the pad ones over the GMASA time and separation between the two becomes more important after the appearance of impact, which starts at $0.21 \mathrm{~s}$. 


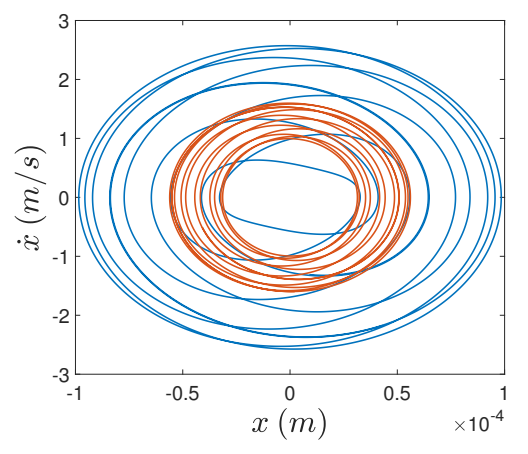

(a) Point D - $\mathrm{x}$ axis

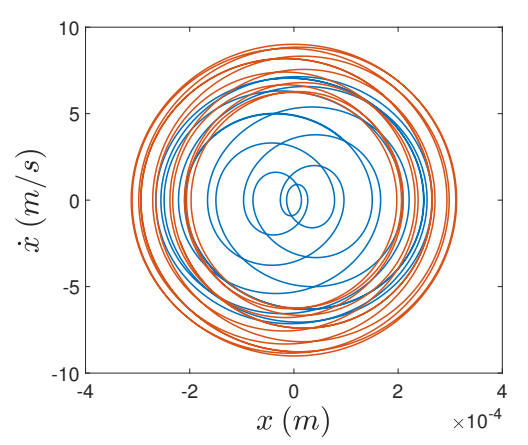

(d) Point E - x axis

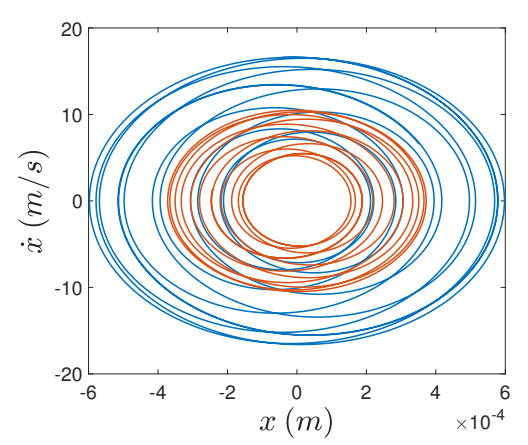

(g) Point $\mathrm{F}-\mathrm{x}$ axis

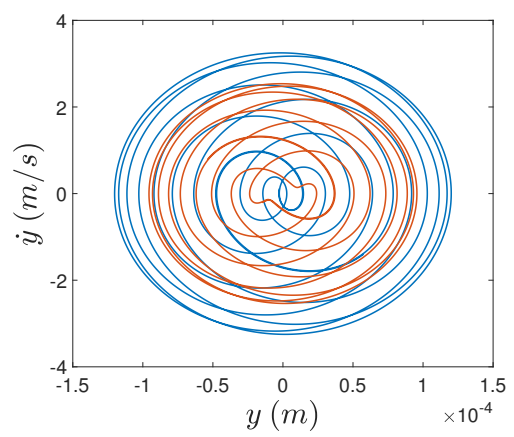

(b) Point D - y axis

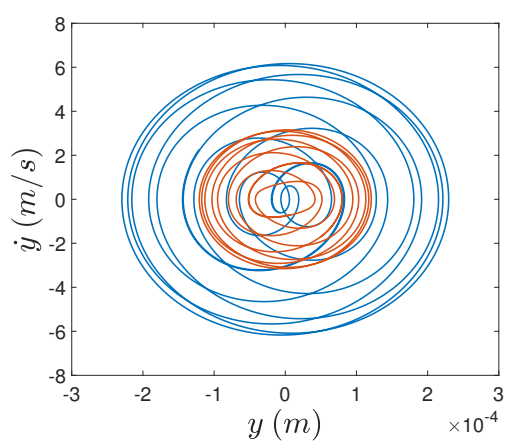

(e) Point E - y axis

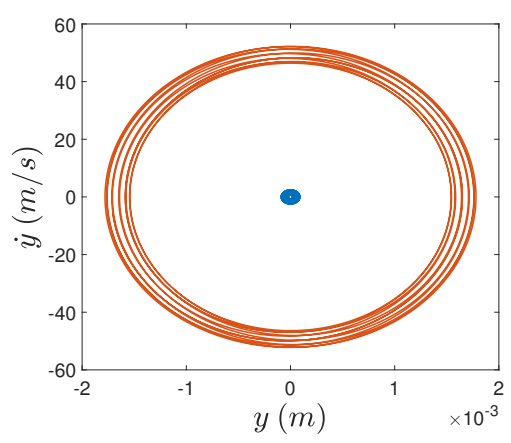

(h) Point F - y axis

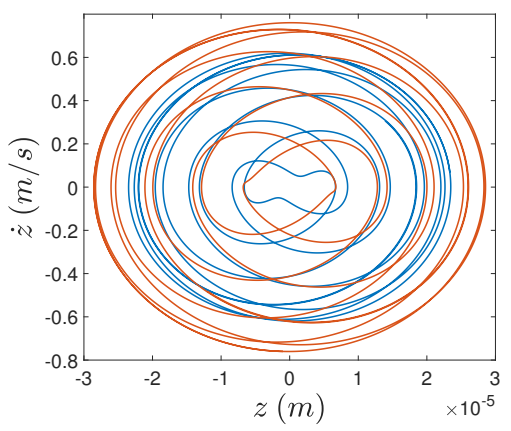

(c) Point D - z axis

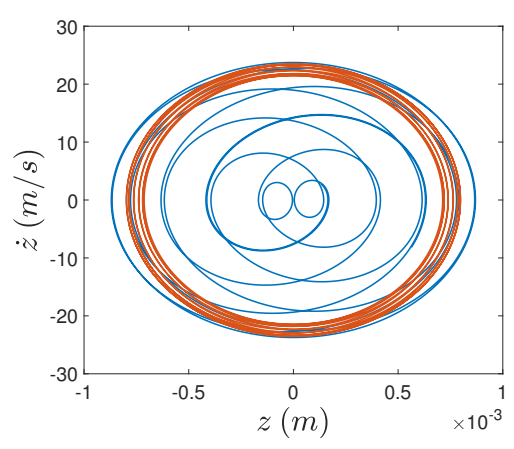

(f) Point E - z axis

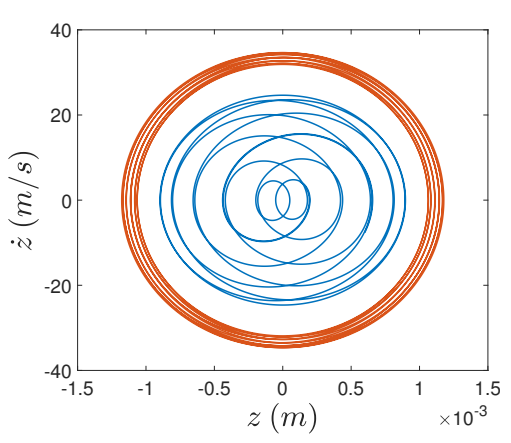

(i) Point F - z axis

Figure 10: Disc torus motions (blue) and pad torus motions (orange) of the points D, E and F of the outer pad

As a conclusion, the evolution of the quasi-periodic orbits is complex and different behaviours are observed depending on the considered point and the considered direction. The orbits start from a simple shape, due to the presence of a unique unstable mode in the dynamic response, but the final orbits present complex behaviours due to the presence of a second unstable mode in the nonlinear dynamic response and the potential coexistence between these two unstable modes.

\section{Conclusions}

In the present work, the nonlinear dynamic behaviour of a full finite element model of an automotive brake system has been computed with the Generalised Modal Amplitude Stability Analysis. For a given friction coefficient, the GMASA was able to counterbalance the under-predictive aspect of the CEA. Indeed, the latter predicted only one unstable mode, when the GMASA captures the emergence of a second mode in the nonlinear dynamic response. The emergence of this second mode is due to the coupling between the two modes due to the nonlinear forces. The analysis of the evolution of the contact conditions at the pad/disc interface show that the amplitude of the oscillations is stabilised by the appearance of micro-impacts. The analysis of the evolution of the torus motions over time show that complex behaviours appear at the pad/disc interface. The study 
demonstrates the capacity of the GMASA approach to predict the unstable vibration modes for large industrial FEM, and more particularly to counterbalance the under-predictive aspect of the CEA.

For future work, a comparison with experiments is expected to ensure the good capacity of the approach to validate experimentally the numerical approach. Moreover, an extensive study about the influence of the the friction contact law, of the braking pressure and of structural modifications on the nonlinear dynamic behaviour of the brake is also planned to account for the uncertainties present in the modelling. Finally, the GMASA is only able to predict periodic or quasi-periodic solutions (i.e. the estimation of more than quasi-periodic motion are not feasible with the actual development). However, its computational efficiency makes it usable for future optimisation campaigns of industrial brakes for the prediction of instability and friction-indcued vibrations and noise.

\section{Acknowledgements}

This work was achieved within PSA Peugeot Citroën Stellab program - OpenLab Vibro-Acoustic- Tribology@Lyon (VAT@Lyon).

J.-J. Sinou acknowledges the support of the Institut Universitaire de France.

\section{References}

[1] D. Majcherczak, P. Dufrenoy, and Y. Berthier. Tribological, thermal and mechanical coupling aspects of the dry sliding contact. Tribology International, 40(5):834-843, 2007.

[2] AR Mat Lazim, M Kchaou, MK Abdul Hamid, and AR Abu Bakar. Squealing characteristics of worn brake pads due to silica sand embedment into their friction layers. Wear, 358:123-136, 2016.

[3] LY Barros, PD Neis, NF Ferreira, RP Pavlak, D Masotti, LT Matozo, Jacob Sukumaran, Patrick De Baets, and M Andó. Morphological analysis of pad-disc system during braking operations. Wear, 352:112-121, 2016.

[4] M Kchaou, AR Mat Lazim, MK Abdul Hamid, and AR Abu Bakar. Experimental studies of friction-induced brake squeal: influence of environmental sand particles in the interface brake pad-disc. Tribology International, 110:307-317, 2017.

[5] Z.Y. Xiang, J.L. Mo, H. Ouyang, F. Massi, B. Tang, and Z.R. Zhou. Contact behaviour and vibrational response of a high-speed train brake friction block. Tribology International, 152:106540, 2020.

[6] Z.Y. Xiang, W. Chen, J.L. Mo, Q.A. Liu, Z.Y. Fan, and Z.R. Zhou. The effects of the friction block shape on the tribological and dynamical behaviours of high-speed train brakes. International Journal of Mechanical Sciences, 194:106184, 2021.

[7] A. Papinniemi, JCS Lai, J. Zhao, and L. Loader. Brake squeal: a literature review. Applied acoustics, 63(4):391-400, 2002.

[8] NM Kinkaid, O. M O'Reilly, and P. Papadopoulos. Automotive disc brake squeal. Journal of sound and vibration, 267(1):105$166,2003$.

[9] H. Ouyang, W. Nack, Y. Yuan, and F. Chen. Numerical analysis of automotive disc brake squeal: a review. International Journal of Vehicle Noise and Vibration, 1(3-4):207-231, 2005.

[10] X. Lorang, F. Foy-Margiocchi, Q. S. Nguyen, and P-E. Gautier. Tgv disc brake squeal. Journal of Sound and Vibration, 293(3-5):735-746, 2006.

[11] F. Massi, L. Baillet, O. Giannini, and A. Sestieri. Brake squeal: linear and nonlinear numerical approaches. Mechanical Systems and Signal Processing, 21(6):2374-2393, 2007.

[12] X Lorang and O Chiello. Stability and transient analysis in the modelling of railway disc brake squeal. In Noise and vibration mitigation for rail transportation systems, pages 447-453. Springer, 2008.

[13] T Butlin and J Woodhouse. A systematic experimental study of squeal initiation. Journal of Sound and Vibration, 330(21):5077-5095, 2011.

[14] S. Oberst and JCS Lai. Statistical analysis of brake squeal noise. Journal of Sound and Vibration, 330(12):2978-2994, 2011.

[15] J-J Sinou, A. Loyer, O. Chiello, G. Mogenier, X. Lorang, F. Cocheteux, and S. Bellaj. A global strategy based on experiments and simulations for squeal prediction on industrial railway brakes. Journal of Sound and Vibration, 332(20):5068-5085, 2013.

[16] T Butlin and J Woodhouse. Friction-induced vibration: Model development and comparison with large-scale experimental tests. Journal of Sound and Vibration, 332(21):5302-5321, 2013.

[17] M. Stender, S. Oberst, M. Tiedemann, and N. Hoffmann. Complex machine dynamics: systematic recurrence quantification analysis of disk brake vibration data. Nonlinear Dynamics, 97(4):2483-2497, 2019.

[18] M. Stender, M. Tiedemann, D. Spieler, D. Schoepflin, N. Hoffmann, and S. Oberst. Deep learning for brake squeal: Brake noise detection, characterization and prediction. Mechanical Systems and Signal Processing, 149:107181, 2021.

[19] RA Ibrahim. Friction-induced vibration, chatter, squeal, and chaos-part i: mechanics of contact and friction. 1994.

[20] A. Akay. Acoustics of friction. The Journal of the Acoustical Society of America, 111(4):1525-1548, 2002.

[21] M Eriksson, A Lundqvist, and S Jacobson. A study of the influence of humidity on the friction and squeal generation of automotive brake pads. Proceedings of the Institution of Mechanical Engineers, Part D: Journal of Automobile Engineering, $215(3): 329-342,2001$.

[22] F. Bergman, M. Eriksson, and S. Jacobson. Influence of disc topography on generation of brake squeal. Wear, 225:621-628, 1999.

[23] M. Eriksson, F. Bergman, and S. Jacobson. Surface characterisation of brake pads after running under silent and squealing conditions. Wear, 232(2):163-167, 1999.

[24] E Denimal, J-J Sinou, S Nacivet, and L Nechak. Squeal analysis based on the effect and determination of the most influential contacts between the different components of an automotive brake system. International Journal of Mechanical Sciences, $151: 192-213,2019$.

[25] T. Butlin and Jim Woodhouse. Sensitivity studies of friction-induced vibration. International journal of vehicle design, 51(1-2):238-257, 2009. 
[26] T Butlin and J Woodhouse. Sensitivity of friction-induced vibration in idealised systems. Journal of Sound and Vibration, 319(1-2):182-198, 2009.

[27] A. Culla and F. Massi. Uncertainty model for contact instability prediction. The Journal of the Acoustical Society of America, 126(3):1111-1119, 2009.

[28] T Butlin and J Woodhouse. Friction-induced vibration: Quantifying sensitivity and uncertainty. Journal of Sound and Vibration, 329(5):509-526, 2010.

[29] T Tison, A Heussaff, F Massa, I Turpin, and RF Nunes. Improvement in the predictivity of squeal simulations: Uncertainty and robustness. Journal of Sound and Vibration, 333(15):3394-3412, 2014.

[30] A. Nobari, H. Ouyang, and P. Bannister. Uncertainty quantification of squeal instability via surrogate modelling. Mechanical Systems and Signal Processing, 60:887-908, 2015.

[31] A Renault, F Massa, B Lallemand, and T Tison. Experimental investigations for uncertainty quantification in brake squeal analysis. Journal of Sound and Vibration, 367:37-55, 2016.

[32] S Oberst and JCS Lai. Nonlinear transient and chaotic interactions in disc brake squeal. Journal of Sound and Vibration, 342:272-289, 2015

[33] S. Oberst and JCS Lai. Chaos in brake squeal noise. Journal of Sound and Vibration, 330(5):955-975, 2011.

[34] BA Wernitz and NP Hoffmann. Recurrence analysis and phase space reconstruction of irregular vibration in friction brakes: Signatures of chaos in steady sliding. Journal of Sound and Vibration, 331(16):3887-3896, 2012.

[35] P Gdaniec, C Weiß, and NP Hoffmann. On chaotic friction induced vibration due to rate dependent friction. Mechanics Research Communications, 37(1):92-95, 2010.

[36] N. K Vitanov, N. Hoffmann, and B. Wernitz. Nonlinear time series analysis of vibration data from a friction brake: Ssa, pca, and mfdfa. Chaos, Solitons \&6 Fractals, 69:90-99, 2014.

[37] S Oberst, JCS Lai, and S Marburg. Guidelines for numerical vibration and acoustic analysis of disc brake squeal using simple models of brake systems. Journal of Sound and Vibration, 332(9):2284-2299, 2013.

[38] K. Soobbarayen, S. Besset, and J-.J. Sinou. A simplified approach for the calculation of acoustic emission in the case of friction-induced noise and vibration. Mechanical Systems and Signal Processing, 50-51:732-756, 2015.

[39] S. Oberst and J.C.S. Lai. Squeal noise in simple numerical brake models. Journal of Sound and Vibration, 352:129-141, 2015.

[40] G. Corradi, J.-J. Sinou, and S. Besset. Performances of the double modal synthesis for the prediction of the transient selfsustained vibration and squeal noise. Applied Acoustics, 175:107807, 2021.

[41] AR AbuBakar and H Ouyang. Complex eigenvalue analysis and dynamic transient analysis in predicting disc brake squeal. International Journal of Vehicle Noise and Vibration, 2(2):143-155, 2006.

[42] G Vermot Des Roches. Frequency and time simulation of squeal instabilities. Application to the design of industrial automotive brakes. PhD thesis, 2011.

[43] N. Coudeyras, J-J Sinou, and S. Nacivet. A new treatment for predicting the self-excited vibrations of nonlinear systems with frictional interfaces: The constrained harmonic balance method, with application to disc brake squeal. Journal of sound and vibration, 319(3-5):1175-1199, 2009.

[44] L Charroyer, O Chiello, and J-J Sinou. Self-excited vibrations of a non-smooth contact dynamical system with planar friction based on the shooting method. International Journal of Mechanical Sciences, 144:90-101, 2018.

[45] J Brunetti, F Massi, W D'Ambrogio, and Y Berthier. A new instability index for unstable mode selection in squeal prediction by complex eigenvalue analysis. Journal of Sound and Vibration, 377:106-122, 2016.

[46] S Nacivet and J-J Sinou. Modal amplitude stability analysis and its application to brake squeal. Applied Acoustics, 116:127$138,2017$.

[47] E Denimal, J-J Sinou, and S Nacivet. Generalized modal amplitude stability analysis for the prediction of the nonlinear dynamic response of mechanical systems subjected to friction-induced vibrations. Nonlinear Dynamics, pages 1-24, 2020.

[48] O Fazio, S Nacivet, and J-J Sinou. Reduction strategy for a brake system with local frictional non-linearities-application for the prediction of unstable vibration modes. Applied Acoustics, 91:12-24, 2015. 


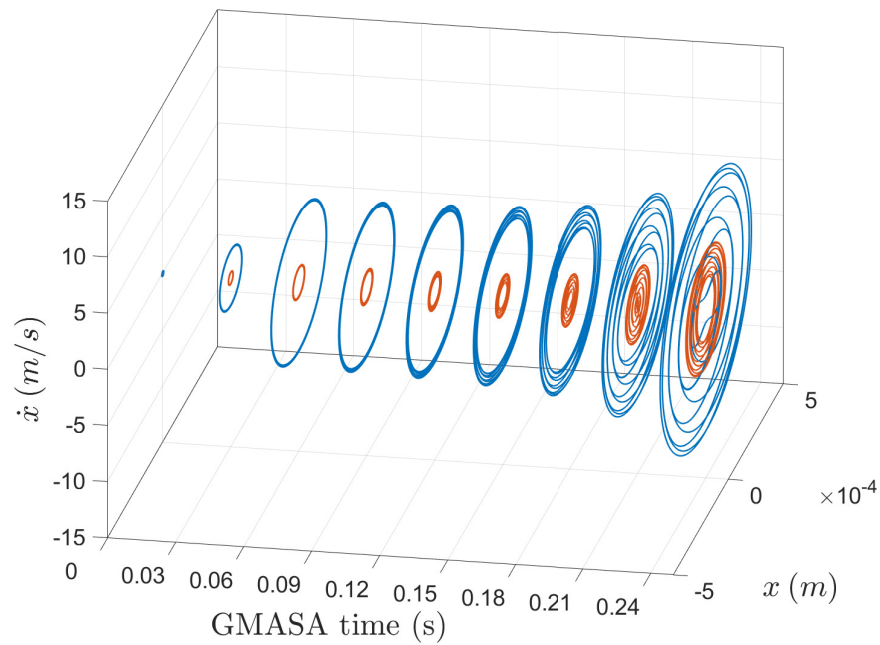

(a) Point $\mathrm{C}-\mathrm{x}$ axis

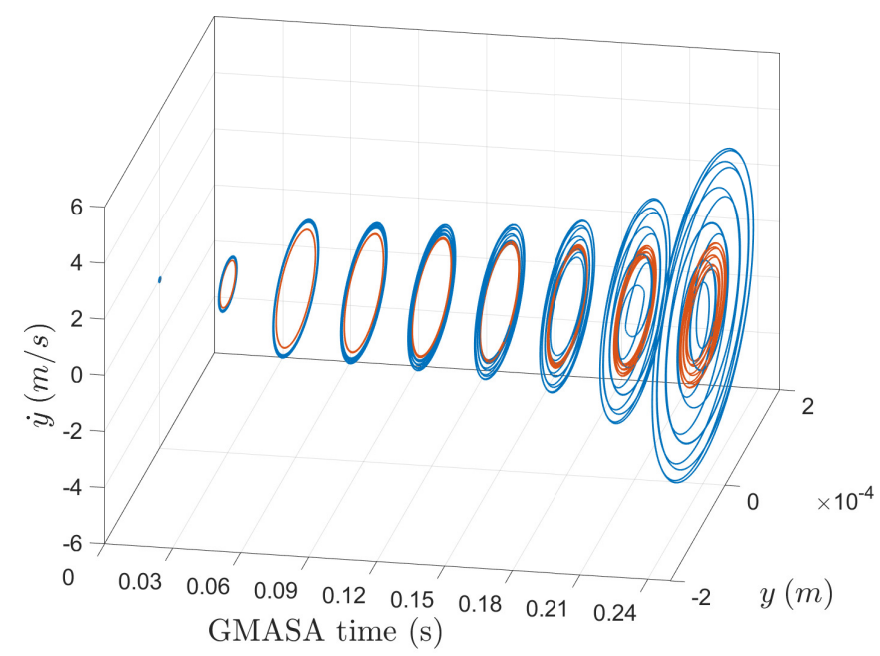

(c) Point $\mathrm{C}-\mathrm{y}$ axis

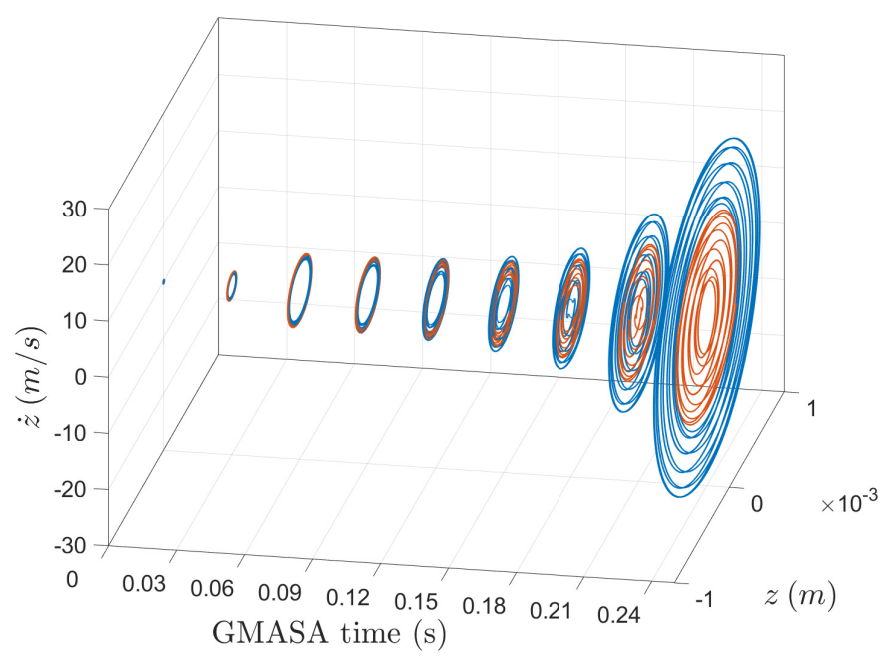

(e) Point $\mathrm{C}-\mathrm{z}$ axis

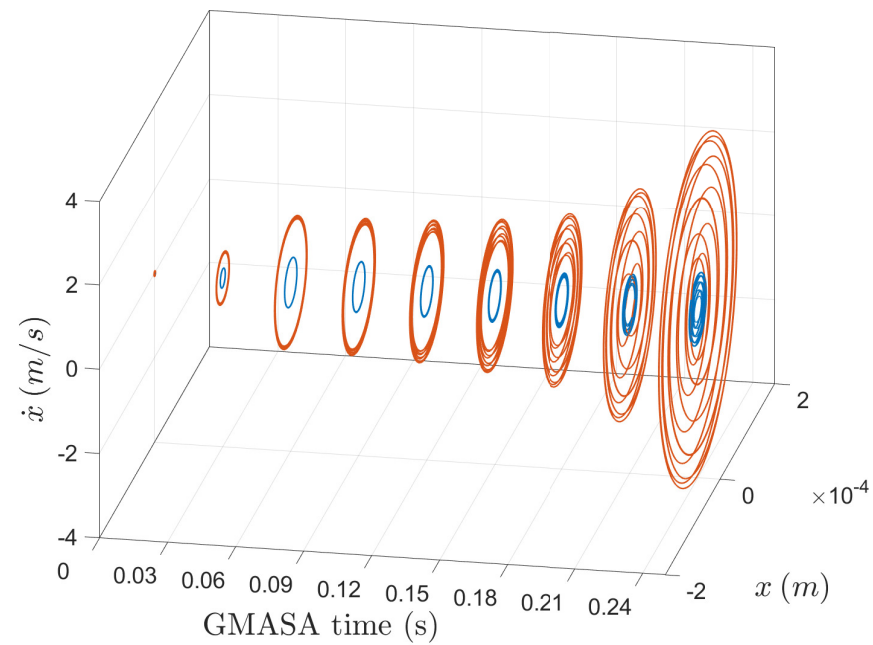

(b) Point $\mathrm{G}-\mathrm{x}$ axis

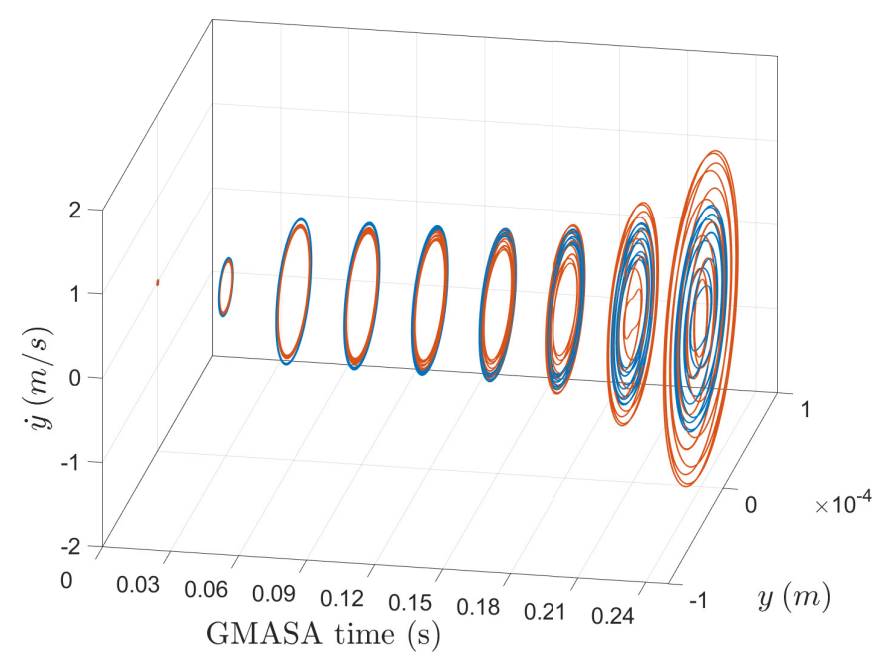

(d) Point G - y axis

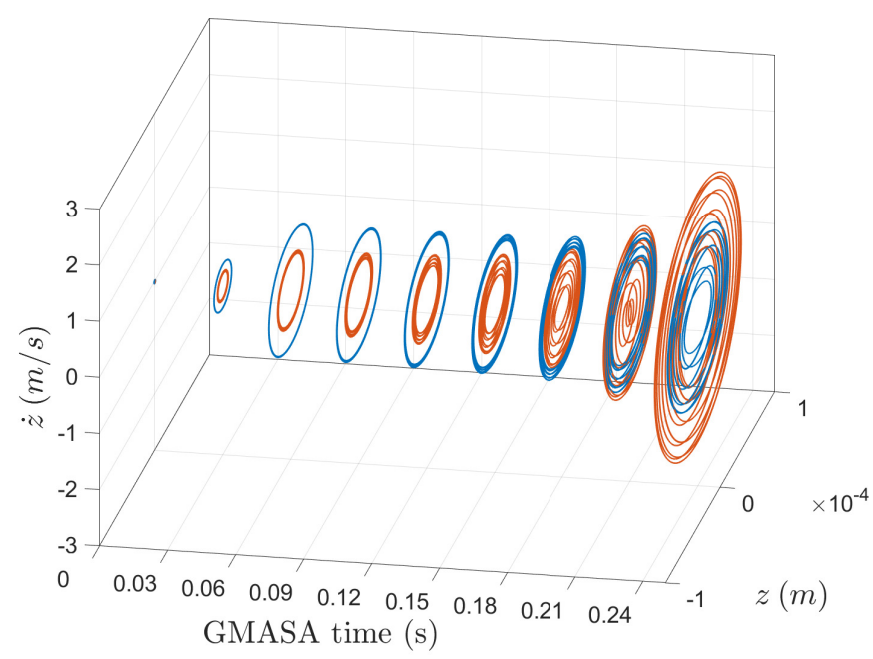

(f) Point $G-z$ axis

Figure 11: Evolution of the disc torus motions (blue) and pad torus motions (orange) over the GMASA time of the points $\mathrm{C}$ (left) and $\mathrm{G}$ (right) 


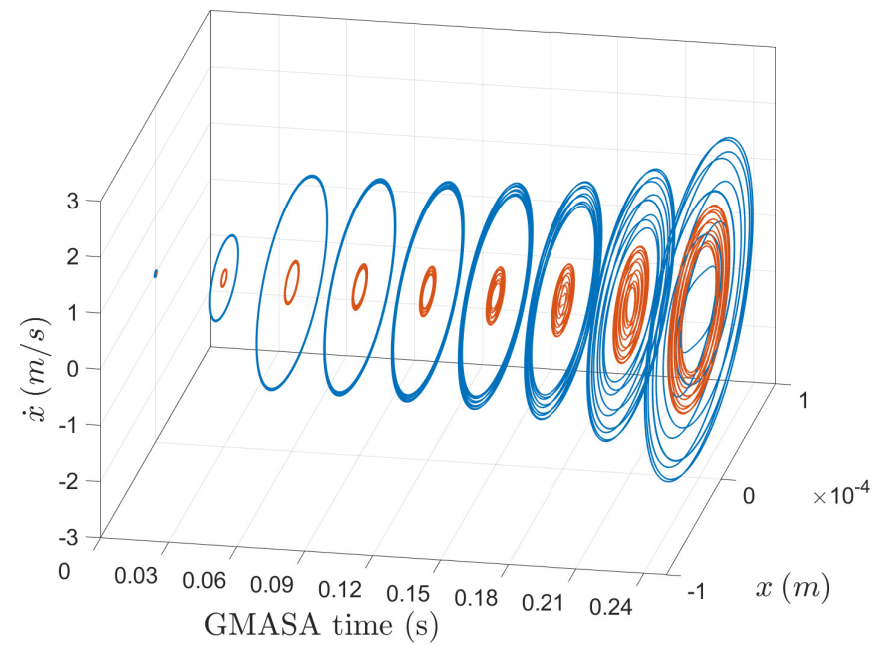

(a) Point D - x axis

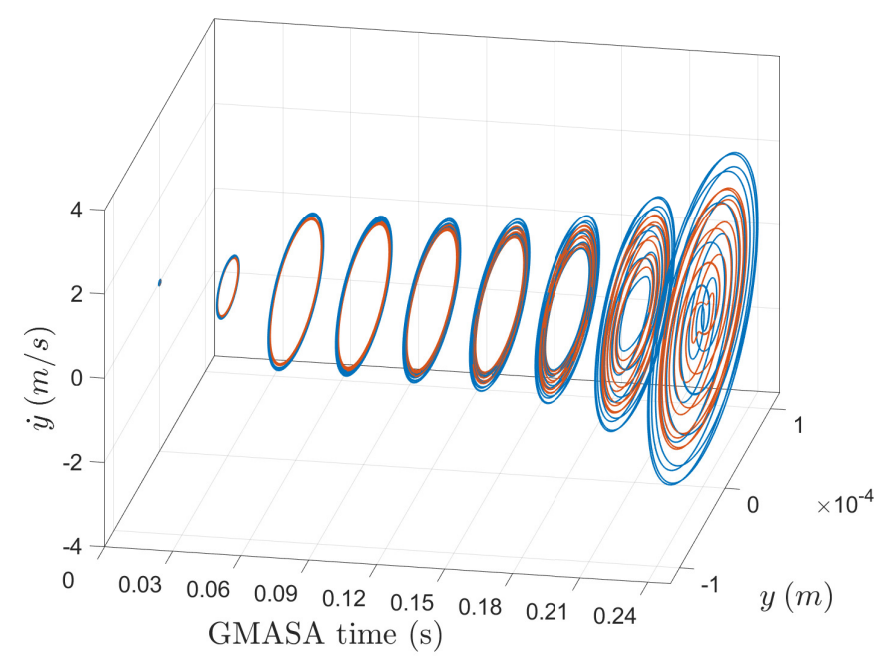

(c) Point D - y axis

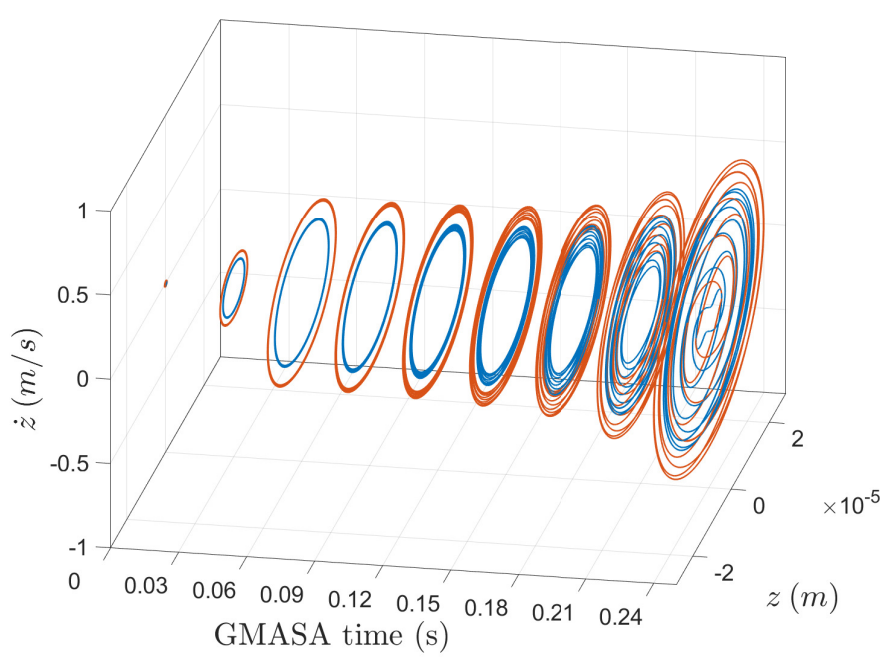

(e) Point D - z axis

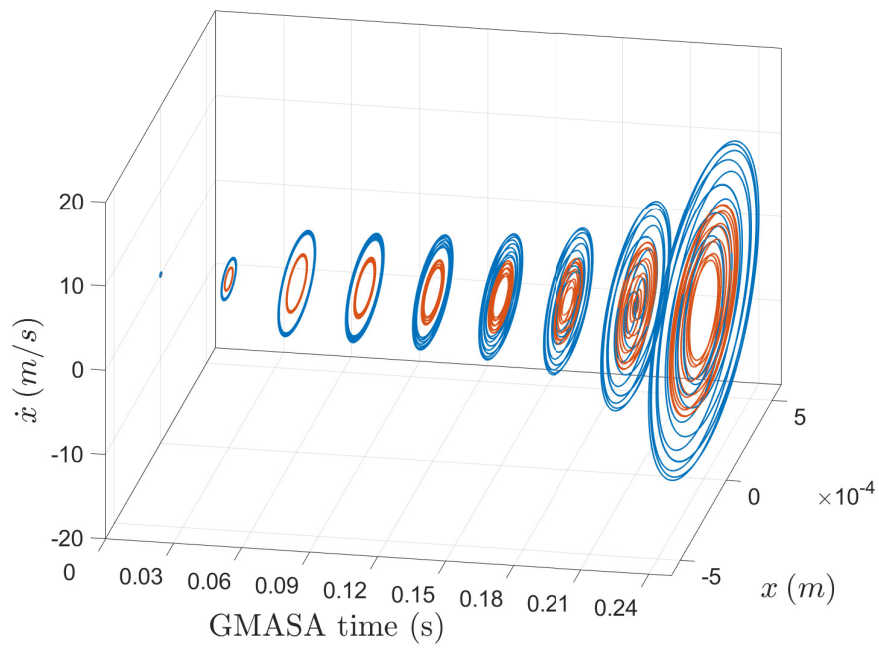

(b) Point $\mathrm{F}-\mathrm{x}$ axis

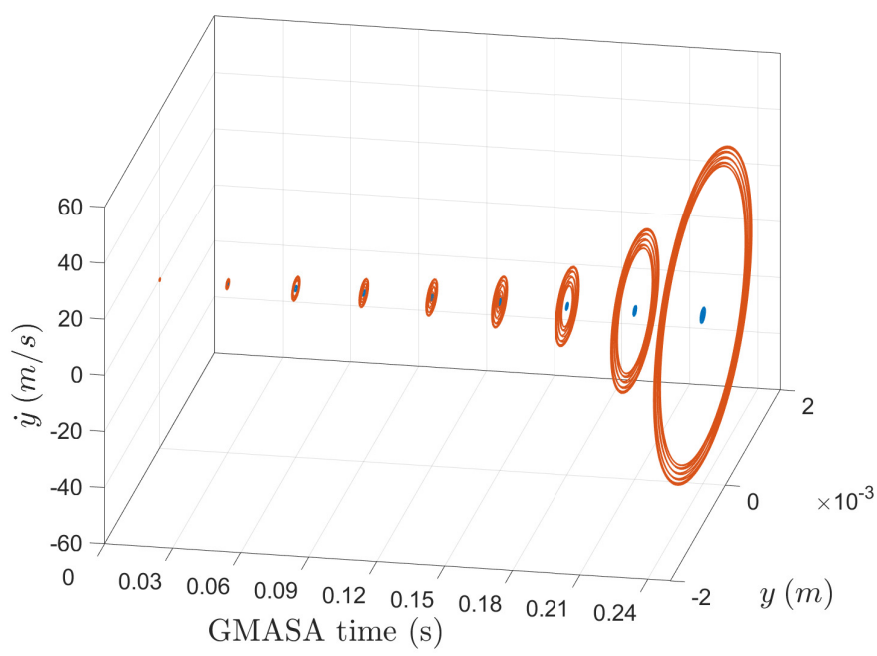

(d) Point F - y axis

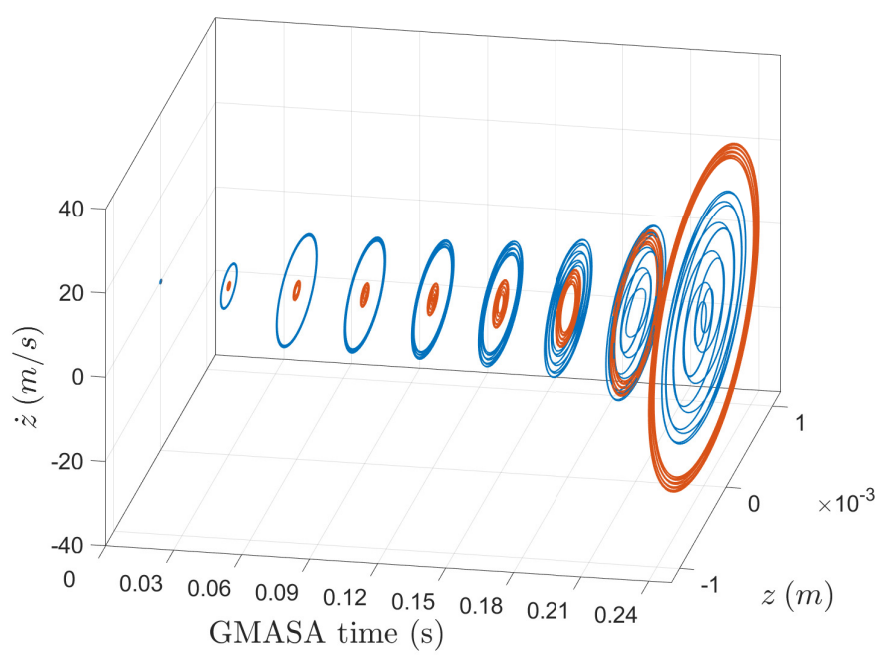

(f) Point $F-z$ axis

Figure 12: Evolution of the disc torus motions (blue) and pad torus motions (orange) over the GMASA time of the points D (left) and F (right) 